\title{
1 Buoyancy flows and pollutant dispersion through different scale urban \\ 2 areas: CFD simulations and wind-tunnel measurements
}

3 Peng-Yi Cui ${ }^{\mathrm{a}}$, Zhuo Li ${ }^{\mathrm{a}}$, Wen-Quan Tao ${ }^{\mathrm{a}, \mathrm{b}, *}$

9 Abstract

10 In this paper, a coupled CFD model was established to study the multiscale problems

11 on the mixed force and buoyancy flow and dispersion passing neighborhood scale - street

12 scale - indoor scale models, and the numerical results were validated by wind-tunnel

13 measurements with Richardson numbers $(\mathrm{Ri})$ from 0 to 4.77 with $\mathrm{SF}_{6}$ being the tracer gas.

14 The basic flow, heat and pollutant transfer were solved with the 3-D steady RANS

15 (Reynolds-Averaged Navier-Stokes) equations. The results show that when $R i \leq 0.85$, the

16 standard $k-\varepsilon$ model (SKE) can better predict the flow and temperature fields. When $R i>$

170.85 , the realizable $k-\varepsilon$ model (RLKE) perform better. For the same turbulence model

18 equation, with the increasing $R i$ the effects of the two near-wall functions (standard and

19 non-equilibrium wall functions, SWFs and NEWFs) on the flow structures and

20 temperature distributions become more and more significant. The specific value of $S c_{t}$

21 has a significant effect on predicting the pollutant dispersion and the optimal value is 0.7

22 for the studied cases. It is also found that for indoor flow caused by an outdoor street flow

23 there also exists the $R e$-independence region. For the model studied only when $R e_{H} \geq$

24 7.57E+03, the wind-tunnel measured results can represent the realistic cases 
25 meaningfully for both flow and pollutant distributions in street canyon and inside the 26 room.

27 Keywords: Wind tunnel; Computational fluid dynamics (CFD); Buoyancy flow; 28 Pollutant dispersion; Multiscale problem

\section{1. Introduction}

31 The physical phenomena that mixed forced and buoyancy flow with harmful 32 pollutants passes over different geometry scale urban areas and enters into indoor 33 environment to affect the health of residents belong to multiscale physical problems [1,2].

34 Recently, the frequent haze weather caused by the increasing level of air pollutants such 35 as $\mathrm{PM}_{10}$ and $\mathrm{PM}_{2.5}$ has caught many people's attentions in many big cities of China, 36 which is mainly resulted from the coal combustion, industrial and traffic exhausts [3-6].

37 Besides, the city managers and planners also pay attentions to the diffusion of air 38 pollutants due to incidents such as terrorist attack and accidental leakage of toxicity fluids

39 in different scale urban areas $[7,8]$. The studied regions can be divided into several ranges

40 of length scales: regional scale (up to 100 or $200 \mathrm{~km}$ ), city scale (up to 10 or $20 \mathrm{~km}$ ), 41 neighborhood scale (up to 1 or $2 \mathrm{~km}$ ) and street scale (less than $100 \sim 200 \mathrm{~m}$ ) [9], indoor 42 scale (less than $10 \sim 20 \mathrm{~m}$ ) and even body scale (less than $1 \sim 2 \mathrm{~m}$ ). However, most of the 43 previous studies mainly concentrated on the same geometry scales, and three approaches

44 have been used: (1) on-site full-scale experiments; (2) reduced-scale physical modeling 45 (wind-tunnel or water-channel experiments); and (3) numerical simulations based on 46 CFD techniques. 
47 There are pros and cons to these three research methods for studying flow and

48 dispersion within urban environment. CFD method can provide detailed information

49 under well-controlled conditions, but the model and method should be validated by some

50 test results. Reduced-scale model can give some accurate experimental data, but is

51 required to overcome the similarity constraints, especially when the forced flow and

52 buoyancy effects are combined together. More intuitive and real data can be obtained

53 from on-site experiments. However, for this method, apart from huge sources required,

54 the experimental repeatability is quite small due to the uncontrollability of the

55 meteorological conditions. van Hoof and Blocken [10] carried out the full-scale

56 measurements of indoor environmental conditions and natural ventilation in a large semi-

57 enclosed stadium and discussed four issues: (1) the advantage and disadvantage of full-

58 scale on-site measurements versus reduced-scale wind tunnel measurements; (2) the

59 limited number of measurement positions inside the stadium; (3) the repeatability of the

60 full-scale measurements; and (4) the possibilities and limitations of the full-scale

61 measurements for CFD validation. Given the above factors, most researchers usually

62 conducted their studies by coupling CFD methods with other two methods for the CFD

63 validation. Gousseau et al. [11] evaluated the performance of two different modeling

64 methods (RANS standard $k-\varepsilon$ model and LES) applied to predict near-field pollutant

65 dispersion in an actual urban environment: downtown Montreal, validated by the detailed

66 wind-tunnel experiments. They found that LES with the dynamic subgrid-scale model

67 shows a better performance without requiring any parameter input to solve the dispersion

68 equation. Allegrini et al. [12] used the wind-tunnel measurements to validate the scaled

69 CFD model for the mixed buoyancy flow in urban street canyons, and the numerical

70 results showed that a significantly better agreement can be achieved by using a standard 
$71 k-\varepsilon$ model with the NEWFs (non-equilibrium wall functions) than LRNM (low-Reynolds

72 number modeling). Stavrakakis et al. [13] applied CFD method coupled with the on-site

73 building-scale experiments to investigate the natural cross-ventilation and evaluate the

74 thermal comfort inside the building. They concluded that the RNG (renormalization

75 group) $k-\varepsilon$ model performed relatively better, especially for temperature predictions and

76 hence they chosen it for further thermal comfort estimation purposes.

77 Obviously, the above studies mainly focused on one geometric scale (neighborhood 78 scale, street scale or building scale). However, the flow and dispersion within urban

79 environment belong to multiscale phenomena, the geometry scales of which are always

80 across more than three orders of magnitude, taking the pollutant transport in Lujiazui

81 region of Pudong district, Shanghai (see Fig. 1) as an example. The pollutant transport

82 process in such an area could be described as: the pollutants are released into the ambient

83 air in somewhere of the upwind direction and diffuse with the air flows that are affected

84 by many factors, then pass through building groups in the neighborhood scale, and arrive

85 at a high-rise building located in a street canyon, finally enter into a room through the

86 open windows, and affect the health of residents. In general, there are three numerical

87 methods to solve the above multiscale problem to obtain the detailed information in the

88 smallest scale geometry. The first one is called "coupled" CFD simulation or full-scale

89 simulation, in which grid generation and equation solving are conducted simultaneously

90 within the whole computational domain. This method allows the proper calculation of air

91 flow in the smallest scale geometry, but needs a high-resolution grid system and a

92 relatively high computational cost for a large grid number [14]. The second method is

93 called "de-coupled" simulation, in which the computational domain is divided into 
94 several regions according to the different geometry scales, then the separate simulations

95 are conducted, for example, one for the outdoor flow and other for the indoor flow, each

96 in their own computational domain [15-17]. In the outdoor simulation, the information

97 (generally pressure coefficients) is obtained at the closed ventilation openings to be used

98 as the boundary condition for the indoor simulation. The accuracy of this method can

99 easily be compromised due to the simplifications involved. To improve the second

100 approach, another "de-coupled" method called "from top-to-down solution procedure" is

101 proposed with increasing grid fineness $[18,19]$. Both the two "de-coupled" methods

102 require an accurate full-scale simulation to validate and evaluate their simulation results.

103 It is well-known that the wind-tunnel experiment is an important tool to investigate

104 the basic flow, heat and mass transfer within urban environment and can also provide

105 data for the validation of the CFD method. However, to the authors' knowledge, the

106 above complex multiscale problems have been only investigated by on-site full-scale

107 measurements coupled with CFD simulations by van Hooff and Blocken [14] and not yet

108 performed by reduced-scale wind tunnel measurements. Their study presented a coupled

109 CFD modeling method for urban wind flow and indoor natural ventilation on a high-

110 resolution body-fitted grid using realizable $k-\varepsilon$ model and the CFD model was validated

111 by the on-site full-scale measurements [14]. Then, they applied this coupled CFD

112 modeling to investigate the effect of direction and urban surroundings on the $\mathrm{ACH}$ of a

113 large semi-enclosed stadium [20]. For the reduced-scale wind-tunnel measurements in

114 order to obtain meaningful results of the multiscale problems mentioned above there are

115 two important challenges. The first challenge is the satisfaction of flow similarity

116 between prototype and wind tunnel. In this regard previous studies [21, 22] have shown 
117 that for flows over buildings once Reynolds number of the oncoming flow is larger than a

118 certain values, denoted by $R e_{c r i t}$, the values of $R e$ will not affect the flow structure too

119 much. The value of $R e_{\text {crit }}$ is usually 2-3 orders of magnitude less than $R e$ of the prototype

120 flow. When $R e$ is larger than $R e_{c r i t}$ flow is in the $R e$-independence region. The existence

121 of $R e$-independence region creates a very favorable condition for adopting small-scale

122 wind tunnel test. Second, it should be guaranteed that the smallest scale geometry of the

123 multiscale modeling satisfies the $R e$-independence requirement. Thus whether the flow in

124 the smallest scale geometry can satisfy $R e$-independence is an important condition for

125 wind-tunnel measurements to reproduce the real flow and dispersion through different

126 scale urban areas.

127 However, most previous studies on the $R e$-independence have been focused on the 128 external flow, such as the flow passing over a single building or building groups [21-23].

129 For the internal flow, such as the flow passing the indoor scale model through the open

130 windows in this study, the concept of Re-independence of the flow structure is no more

131 applicable. The air flow passing through the open windows and entering the rooms will

132 be the turbulent flow in the reality but would be likely laminar flow in wind-tunnel tests.

133 According to the above analysis, when the reduced scale model is used, it is necessary to

134 confirm the flow-structure independence for the studied multiscale models, including the

135 street and indoor scales.

136 The above discussion has set forth the background of the present study. The first aim 137 of the present work is to construct a coupled CFD model for accurately simulating the 138 multiscale problems on the flow and pollutant dispersion within different scale urban 139 areas. The feasibility of the adopted turbulence models are examined by comparing their 
140 predictions with the wind-tunnel measurements, which were reported in our previous

141 work, where a simple multiscale model with three geometry scales (neighborhood scale -

142 street scale - indoor scale) was constructed by considering the thermal effects with the

143 range of $R i$ from 0 to 4.77 [24]. The influence of turbulent Schmidt number $\left(S c_{t}\right)$ on the

144 pollutant dispersion is considered. The second purpose of this study is to clarify whether

145 there exists Re-independence for the flow structures in the scaled indoor environment.

146 The third one is to examine the effects of the outdoor environment on the IAQ (indoor air

147 quality) by numerical and experimental methods.

\section{2. Description of the experiments}

149 The wind-tunnel experiments were completed in TJ-1 and TJ-4 wind-tunnel

150 laboratories (The State Key Laboratory of Civil Engineering for Disaster Prevention,

151 Tongji University, Shanghai). To model the flow and pollutant dispersion within the

152 different scale urban areas, a simple multiscale physical model is designed with a scale

153 ratio of 1:100 (Fig. 2). This model can be divided into three different scales: (a)

154 neighborhood scale; (b) street scale and (c) indoor scale. Their detailed dimensions are

155 shown in Fig. 2, where $L$ is the characteristic height equal to $0.16 \mathrm{~m}$. The pollutant source

156 with dimensions of $2 \mathrm{~cm} \times 2 \mathrm{~cm} \times 2 \mathrm{~cm}$ is located on the rooftop of the low-rise building

157 and $\mathrm{SF}_{6}$ is used as a tracer gas and released from the top surface of the pollutant source

158 with a constant release rate of $0.8 \mathrm{~L} / \mathrm{min}$. The ground between the two buildings is heated

159 by rubber heating mats with dimensions of $L \times L$ to model the solar radiation. According

160 to the nondimensionalization of the governing equations by Snyder [22], for an accurate

161 modeling, several dimensionless parameters in the prototype must be duplicated in the 
162 wind-tunnel experiments. The first important dimensionless parameter is Reynolds

163 number $(R e)$, which represents the ratio of inertial and viscous forces, defined as:

$164 \quad \operatorname{Re}=\frac{U_{R} H}{v}$

165 where $U_{R}$ represents the reference velocity $(\mathrm{m} / \mathrm{s})$ which is the free-stream velocity in this

166 study. $H$ is the building height $(\mathrm{m})$ and $v$ is the kinematic viscosity of the air $\left(\mathrm{m}^{2} / \mathrm{s}\right)$. The

$167 R e$ here is called building height Reynolds number $\left(R e_{H}\right)$. To consider the effect of the

168 buoyancy force induced by the ground heating on the flow fields, Richardson number $(R i)$

169 is used, defined as:

$170 \quad R i=\frac{G r}{R^{2}}=\frac{g \beta\left(T_{w}-T_{r e f}\right) H^{3}}{v^{2}} \frac{v^{2}}{U_{R}^{2} H^{2}}=\frac{g \beta\left(T_{w}-T_{r e f}\right) H}{U_{R}^{2}}$

171 where $G r$ is Grashof number, $T_{w}$ is the surface temperature of the heated ground (K), $T_{r e f}$

172 is the ambient temperature $(\mathrm{K}), g$ is the gravitational acceleration $\left(\mathrm{m} / \mathrm{s}^{2}\right)$ and $\beta$ is the

173 volumtric expansion coefficient $(1 / \mathrm{K})$.

174 The wind-tunnel experiments were run at the mean streamwise velocities from 0.4 to 6

$175 \mathrm{~m} / \mathrm{s}$ coupled with the temperature of the heated ground from 15 to $200{ }^{\circ} \mathrm{C}$, which

176 corresponded to several cases with a wide range of $R i$ from 0 to 4.77 (shown in Table 1).

177 The inlet profiles at the inlet plane (Fig. 2(a)) were measured by 3-D Cobra probe with

178 the accuracy of $\pm 0.1 \mathrm{~m} / \mathrm{s}$, which can be used to provide realistic inlet conditions for the

179 numerical simulations. PIV, T-type thermocouples and $\mathrm{SF}_{6}$ quantitative detector were

180 used to measure flow fields, temperature and pollutant distributions [24]. 


\subsection{Governing equation}

183 The RANS turbulence models can provide reasonable solutions for a wide range of 184 problems on the basic flow, heat and pollutant transfer while requiring relatively low 185 computational cost. The generation of buoyancy force due to the thermal effect between

186 the two building models was taken into account in this work, and the governing equations

187 include: the continuity equation in incompressible form,

$188 \quad \frac{\partial u_{j}}{\partial x_{j}}=0$

189 the RANS equation,

$190 \frac{\partial\left(u_{j} u_{i}\right)}{\partial x_{j}}=\frac{\partial}{\partial x_{j}}\left(\left(\mu+\mu_{t}\right) \frac{\partial u_{i}}{\partial x_{j}}\right)-\frac{1}{\rho} \frac{\partial p}{\partial x_{i}}+g_{i}$

191 the energy equation,

$192 \frac{\partial\left(u_{j} T\right)}{\partial x_{j}}=\frac{\partial}{\partial x_{j}}\left(\left(\frac{\mu}{P r}+\frac{\mu_{t}}{\sigma_{t}}\right) \frac{\partial T}{\partial x_{j}}\right)+S_{T}$

193 and a transport equation for a passive advection-diffusion,

$194 \frac{\partial\left(\rho u_{j} c_{i}\right)}{\partial x_{j}}=\frac{\partial}{\partial x_{j}}\left(\left(\rho D_{i, m}+\frac{\mu_{t}}{S c_{t}}\right) \frac{\partial c_{i}}{\partial x_{j}}\right)+S_{i}$

195 where $u_{i}$ is the time-averaged velocity $(\mathrm{m} / \mathrm{s}) ; u_{j}$ is the velocity vector $(\mathrm{m} / \mathrm{s}) ; \mu$ is the 196 molecular viscosity $(\mathrm{Pa} \cdot \mathrm{s}), \mu_{t}$ is the turbulent viscosity $(\mathrm{Pa} \cdot \mathrm{s}) ; p$ is the pressure $(\mathrm{Pa}), g_{i}$ is

197 the gravitational body force $\left(\mathrm{m} / \mathrm{s}^{2}\right), \rho$ is the density $\left(\mathrm{kg} / \mathrm{m}^{3}\right) ; T$ is the temperature $(\mathrm{K}) ; P r$

198 is Prandtl number; $\sigma_{t}$ is the turbulent Prandtl number (also written as Prt $=0.85$ ); $S_{T}$ is the

199 heat source term $\left(\mathrm{W} /\left(\mathrm{m}^{3} \cdot \mathrm{s}\right)\right) ; c_{i}$ is the local mass concentration of $\mathrm{SF}_{6}\left(\mathrm{~kg} / \mathrm{m}^{3}\right) ; D_{i, m}$ is the

200 diffusion coefficient for $\mathrm{SF}_{6}\left(\mathrm{~m}^{2} / \mathrm{s}\right) ; S_{i}$ denotes the pollution source term $\left(\mathrm{kg} /\left(\mathrm{m}^{3} \cdot \mathrm{s}\right)\right) ; S c_{t}$

201 is the turbulent Schimdt number which are adopted to consider the effect on $\mathrm{SF}_{6}$ 202 dispersion. 
The standard $k-\varepsilon$ model (SKE) and realizable $k-\varepsilon$ model (RLKE) with the standard

204 wall functions (SWFs) and the non-equilibrium wall functions (NEWFs) for the near-wall

205 treatments were used to model turbulence flow for their good performance in modeling

206 the flow structures [12,14]. Although the low-Reynolds number modeling (LRNM)

207 maybe give more accurate simulation results, it requires a very fine cell close to the wall

208 to resolve the near wall region, which increases the large difficulty of grid generation and

209 computing time cost. Moreover, the grid used by LRNM is unsuitable for the high-

210 Reynolds number models (SKE and RLKE) because the so fine cell close to the wall

211 cannot satisfy the first node near the wall locating out of the viscous sub-layer [12,25].

212 The equations of the two adopted turbulence models are provided in Table 2.

213 The standard wall functions are based on the work of Launder and Spalding [26], and

214 have been worked reasonably well for a broad range of wall-boundary flows. The law-of-

215 the-wall for mean velocity yields,

$216 \quad U^{*}= \begin{cases}y^{*} & \left(y^{*}>11.225\right) \\ \frac{1}{\kappa} \ln \left(E y^{*}\right) & \left(y^{*}<11.225\right)\end{cases}$

217 where

$218 \quad U^{*}=\frac{U_{P} C_{\mu} k_{P}^{1 / 2}}{\tau_{w} / \rho}$

219 is the dimensionless velocity.

$220 \quad y^{*}=\frac{\rho C_{\mu}^{1 / 4} k_{P}^{1 / 2} y_{P}}{\mu}$

221 is the dimensionless distance from the wall and $\kappa$ is von Karman constant $(=0.4187), E$ is

222 the empirical constant $(=9.793), U_{P}$ is the mean velocity of the fluid at the near-wall

223 node $\mathrm{P}, k_{P}$ is the turbulence kinetic energy at the near-wall node $\mathrm{P}, y_{P}$ is the distance from 
224 the point $\mathrm{P}$ to the wall, $\mu$ is the dynamic viscosity of the fluid. The temperature wall

225 functions include the contribution from the viscous heating and for the incompressible

226 flow calculations the law-of-the-wall for the temperature field has the following

227 composite form:

$228 T^{*}=\frac{\left(T_{w}-T_{p}\right)\left(C_{\mu}^{1 / 4} k_{p}^{1 / 2}\right)}{\left(q_{w} / \rho c_{p}\right)}=\left\{\begin{array}{c}\operatorname{Pry}^{*} \quad\left(y^{*}<y_{T}^{*}\right) \\ \operatorname{Pr}_{t}\left[\frac{1}{\kappa} \ln \left(E y^{*}\right)+P\right] \quad\left(y^{*}>y_{T}^{*}\right)\end{array}\right.$

229 where $P$ is given by Jayatilleke [27]:

$230 P=9.24\left(\frac{P r}{P r_{t}}-1\right)\left(\frac{P r}{P r_{t}}\right)^{-1 / 4}$

231 and $y_{T}^{*}$ is the dimensionless thermal sublayer thickness, $c_{p}$ is the specific heat of the fluid,

$232 q_{w}$ the wall heat flux, $T_{p}$ is the temperature at the first near-wall node $\mathrm{P}, T_{w}$ is the

233 temperature at the wall, $P r$ is the molecular Prandtl number $\left(=\mu c_{p} / a, a\right.$ is the coefficient

234 of heat diffusion), $P r_{t}$ is the turbulent Prandtl number (=0.85 at the wall).

235 However, the standard wall functions tend to become less reliable when the flow

236 situations depart from the ideal conditions and are subjected to severe pressure gradients

237 and strong non-equilibrium. The non-equilibrium wall functions are introduced and can

238 potentially improve the results in above situations [28]. The law-of-the-wall for mean

239 temperature remains the same as in the standard wall functions described above. The log-

240 law for mean velocity sensitized to the pressure gradients is

$241 \quad \frac{\tilde{U} C_{\mu}^{1 / 4} k^{1 / 2}}{\tau_{w} / \rho}=\frac{1}{\kappa}\left(E \frac{\rho C_{\mu}^{1 / 4} k^{1 / 2} y}{\mu}\right)$

242 where

$\tilde{U}=U-\frac{1}{2} \frac{d p}{d x}\left[\frac{y_{v}}{\rho \kappa \sqrt{k}} \ln \left(\frac{y}{y_{v}}\right)+\frac{y-y_{v}}{\rho \kappa \sqrt{k}}+\frac{y_{v}^{2}}{\mu}\right]$ 
244 and $y_{v}$ is the physical viscous sublayer thickness, and computed from

$245 \quad y_{v}=\frac{\mu y_{v}^{*}}{\rho C_{\mu}^{1 / 4} k_{P}^{1 / 2}}$

246 where $y_{v}^{*}=11.225$.

247 In this study, Boussinesq model was used to treat the variable air density in which the

248 air density is taken as a constant in all terms of the solved equations, except for the

249 buoyancy term in the momentum equation:

$250 \quad\left(\rho-\rho_{0}\right) g=-\rho_{0} \beta\left(T-T_{0}\right) g$

251 where $\rho_{0}$ is the referenced density of the air flow $\left(\mathrm{kg} / \mathrm{m}^{3}\right) ; T_{0}$ is the referenced temperature

$252(\mathrm{~K})$; Equation (15) is obtained by the Boussinesq approximation $\rho=\rho_{0}(1-\beta \Delta T)$ to

253 replace the buoyancy terms (pressure and gravity terms). This approximation is

254 acceptable as long as changes in actual density are small. Specifically, it is valid when $\beta$

$255\left(T-T_{0}\right)<<1$, and should not be used if the temperature difference in the domain is large.

256 According to the values of $\beta \cdot \Delta T$ shown in Table 1, Cases 2 and 3 are modeled with

257 Boussinesq approximation. For Cases $4 \sim 8$, the incompressible ideal gas law was chosen

258 to define the density, thus the additional equation, Eq. (16), should be solved,

$259 \rho=\frac{M p_{o p}}{R T_{l}}$

260 where $R$ is the universal gas constant $(\mathrm{J} /(\mathrm{mol} \cdot \mathrm{K})), M$ is the molecular weight of the air

$261(\mathrm{~g} / \mathrm{mol}), p_{o p}$ is the operating pressure (pa), and $T_{l}$ is the local temperature $(\mathrm{K})$. Except for

262 density, the specific heat capacity, the thermal conductivity and the viscosity were

263 approximated as a piecewise-liner function of the temperature in the Navier-Stokes 
264 equations. The eight studied cases and their correspondent parameters, including $R i, R e$

265 and $G r$ numbers, are shown in Table 1.

266

267

268

269

270

271

272

273

274

275

276

277

278

279

280

281

282

283

284

285

286

287

\subsection{Computational domain and grid generation}

Since the multiscale building models are symmetrical geometries along the $x$-z plane (Fig. 2), a half of the whole geometry was taken as the computational domain (Fig. 3(a)). Some preliminary simulations of the entire domain were carried out to explore the effects of the size of the computational domain ( $L x, L y$ and $L z$ in Fig. 24(a)), and to ensure the computational domain large enough that the calculation results are independent of the domain. The appropriate computational domain were finally determined as $L x-=L y+=$ $L z+=3 L$ and $L x+=5 L$, where $L$ equals to $0.16 \mathrm{~m}$.

To reduce the computational cost, a non-uniform structured grid system is used to discretize the computational domain. The generated grid system can be described as follows: a grid with the finest resolution of the cell scale of $0.001-0.004 \mathrm{~m}$ is used to discritize the room scale geometry and the rooftop stack, in the street scale a finer grid resolution of $0.004-0.01 \mathrm{~m}$ is used, and the farther the distance from the street, the coarser the grid is applied to discretize the neighborhood scale geometry, where the ratio of two neighborhood cell dimensions is kept from $1.02-1.16 \mathrm{~m}$. Four grid systems with different resolution based on guidelines of Franke et al. [29] are used to examine the grid sensitivity. The grid numbers of this four systems are $6.4 \mathrm{E}+05$ (Grid-1), $8.8 \mathrm{E}+05$ (Grid2), $1.42 \mathrm{E}+06$ (Grid-3) and $2.68 \mathrm{E}+06$ (Grid-4), respectively. The velocity distributions along the room horizontal centerline with the the same numerical setups using the four grid systems are compared in Fig. 4. Obviously, the results from Grid-3 and Grid-4 are fairly close to each other, and the relative deviation of between Grid-3 and Grid-4 is less than $4 \%$, which indicates that the grid resolution of Grid-3 is adequate to guarantee the 
289 to ensure the accuracy for the application of high-Reynolds turbulent model $[25,30]$. In

290 this study, the cases with Grid-3 system had the Y plus values between 11 and 15.

\section{$291 \quad 3.3$ Boundary conditions and computational solution}

292 The measured velocities on the inlet plane are uniform and imposed as the inlet

293 boundary conditions for the numerical model, and the inlet turbulent kinetic energy $(k)$

294 and dissipation $(\varepsilon)$ [31] are defined as,

$k=\frac{3}{2}(\bar{u} I)^{2}$

296 where,

$I=0.16\left(R e_{D}\right)^{-\frac{1}{8}}$

298 where $\bar{u}$ is the mean velocity (m/s), $I$ is the turbulent intensity (\%), $R e_{D}$ is the Reynolds

299 number of the inlet plane based on the hydraulic diameter of the wind-tunnel cross

300 section. The dissipation rate $\varepsilon$ is specified as

$301 \varepsilon=c_{\mu}^{\frac{3}{4}} \frac{k^{\frac{3}{2}}}{l}$

302 where $l$ is the turbulence length scale $(\mathrm{m})$, equals to $0.07 L, L$ is the hydraulic diameter of

303 inlet plane $(L=0.8 \mathrm{~m}), c_{\mu} \approx 0.0845$. The other detailed boundary conditions are

304 summarized in Table 3. For all the simulations, the SIMPLE algorithm was adopted to

305 handle the velocity-pressure coupling and second order upwind scheme was applied to

306 discretize the convective term for a reasonable numerical precision $[25,30]$. The

307 convergence criterion of the iteration is the relative residuals of all dependent variable

308 being less than $10^{-5}$. This is a general practice for examining convergence and well-

309 accepted in similar simulations [32,33]. 


\section{Results and Discussion}

\subsection{Validation for the CFD models}

312 In this section, to validate the CFD models, dimensionless velocity profiles in the

313 street and indoor scales are firstly compared with wind-tunnel measurements qualitatively

314 and quantitatively. The streamlines and temperature distributions in the street canyon for

315 some cases are also used for further validations. Then the effects of $S c_{t}$ on pollutant

316 dispersion are considered to determine a better choice of CFD models. The validated

317 CFD models then are applied to investigate the flow-structure independence in the street

318 and indoor scale models. Finally, the effects of the outdoor environment on the IAQ are

319 studied.

\section{4.1.1. Velocity comparison}

321 As indicated above, the flow studied is the mixed one for which $R i$ is the key 322 parameter. Therefore velocity distributions will be presented for different $R i$ cases. In Fig.

323 5, the numerical results from four turbulence models (SKE-SWFs, SKE-NEWFs, RLKE-

324 SWFs and RLKE-NEWFs) and wind-tunnel measurements are presented for the 325 normalized velocity profiles along the vertical centerline of the street canyon for eight $R i$

326 cases. Some features can be observed. Firstly, from the velocity profiles of wind-tunnel

327 measurements, the minimum velocity (marked with the dotted lines in the figures) in the

328 vertical centerline is moving upwards with the increase of $R i$, which may be ascribed to

329 the upward movement of the vortex center due to the buoyancy effect. Secondly, when $R i$

330 is less than 0.85 (Cases 1-3), for the SKE models with two different wall functions (SKE-

331 SWFs, SKE-NEWFs) there is a good agreement between the test data and the predicted

332 velocity profiles. Thirdly, when $R i$ is equal to or larger than 0.85 (Cases $4-8$ ), the velocity

333 distributions from RLKE-SWFs $(R i=1.22)$ and RLKE-NEWFs $(R i=1.56,4.77)$ have a 
334 better agreement. Thus generally speaking for mixed turbulent flow when Richardson

335 number is small, SKE may be used, while for high $R i$ case RLKE is a better choice. From

336 Fig. 5 one thing is worth noting: Case 5 and Case 6 have the same $R i$ but different $G r$ and

$337 R e$. However, either the measured or the predicted velocity profiles of the two cases are

338 very close to each other, once again indicating that for the mixed flow studied Richardson

339 number is the dependent criteria.

340 Come here to analyze the difference between measured and predicted velocities

341 qualitatively. The correlation coefficient, $R^{2}$, is adopted to investigate the relative

342 agreement between numerical results and wind-tunnel measurements. $R^{2}$ is defined as

343 follows,

$344 \quad R^{2}=\frac{\left(\sum\left(U_{r e f}-\bar{U}_{r e f}\right)\left(U_{c o m}-\bar{U}_{c o m}\right)\right)^{2}}{\sum\left(U_{r e f}-\bar{U}_{r e f}\right)^{2} \sum\left(U_{c o m}-\bar{U}_{c o m}\right)^{2}}$

345 where $U_{\text {ref }}$ is the reference velocity (wind-tunnel results) $(\mathrm{m} / \mathrm{s}), U_{\text {com }}$ is the numerically

346 predicted velocity $(\mathrm{m} / \mathrm{s})$. Obviously for the perfect case $R^{2}$ has its upper limit of 1.0. The

347 closer the value of $U_{\text {com }}$ to $U_{\text {ref, }}$, the greater value of the correlation coefficient. Figure 6

348 compares $R^{2}$ of the dimensionless velocity distributions shown in Fig. 5. It can be seen

349 that for Cases $1-4(R i \leq 0.85)$, the values of $R^{2}$ of SKE-SWFs are all close to 0.9 and

350 larger than other models. With regard to Cases 5 and 6, the calculation results of RLKE-

351 SWFs have a better agreement with the test data than the other models. When $R i>1.22$

352 (Cases 7 and 8), the agreement of results from RLKE-NEWFs with wind-tunnel test data

353 is a bit better. From the above quantitative analysis, it can be found that for the problem

354 studied there is no general turbulence model which can satisfactorily simulate the

355 different cases. Furthermore, it is worth noting that with the increase of $R i$, the values of

$356 R^{2}$ become less and less, which can be attributed to the more and more complex flow 
357 structures caused by the interaction between the inertial force and the increasing 358 buoyancy force generated by the heated ground.

359 In Fig. 7, normalized velocity distributions along the horizontal centerline in the 360 indoor scale (red dotted line in first figure shown in Fig. 7) for different $R i$ cases are 361 compared between wind-tunnel measurements and numerical simulations with different 362 turbulence models. In general, due to the ventilation through the open windows, the 363 indoor flow structures are mainly influenced by the flow structures in the street from the 364 qualitative overview, the matching degree of the indoor velocity profiles between the 365 measured data and numerical simulations is worse than that in the street scale (Fig. 5 vs. 366 Fig. 7), which may indicate that a little difference of the flow structure in the street scale 367 will lead to a large discrepancy for the flow structures in the indoor scale, especially if the 368 window dimension (interface of the two geometry scales) is a small one . However, 369 generally speaking, following feature may be noted. Up to $R i=1.22$, the results of SKE-

370 SWFs have a better agreement compared with others, while when $R i=1.56$ and 4.77 the 371 agreement of RLKE-NEWFs seems better. This situation is quite similar with those 372 obtained for Fig. 5. Figure 8 shows the correlation coefficients $\left(R^{2}\right)$ of the dimensionless 373 velocities in the street scale compared with the indoor scale both calculated by using the 374 models of SKE-SWFs and RLKE-NEWFs. From this figure, we can see that the values of $375 R^{2}$ for both the street scale and the indoor scale (the left column and right column, 376 respectively for each case) are getting smaller with the increase of $R i$. And it is 377 noteworthy that for all the studied cases, the values of $R^{2}$ in the street scale are 378 remarkably larger than that in the indoor scale. Moreover, for Cases 1-4 with the increase 379 of $R i$ the discrepancy of $R^{2}$ between this two geometry scales (the left column $v s$. the right 380 column) becomes larger and larger. From the above findings, it may suggest that even for 
381 the same physical model within different geometry scales, when the air flow from the

382 street scale (a large scale) enters into the indoor scale (a small scale) through an open

383 window, the difference of the flow structures in the street scale simulated by two

384 turbulence models will be amplified in the indoor scale.

385 To obtain a more intuitive comparison, in Fig. 9 the normalized streamlines in the

386 vertical central plane of the street canyon by using PIV measurements are compared with

387 four turbulence models for Cases 1, 4, 5 and 7. For Case 1, there is no buoyancy effect

388 and the flow field is isothermal $(R i=0, R e=6.49 \mathrm{E}+04, G r=0)$. It is remarkable that

389 there is a vortex in the street canyon by PIV measurement, and the simulated results from

390 SKE-SWFs and SKE-NEWFs agree with the measured data much better than those of

391 RLKE-SWFs and RLKE-NEWFs. In addition, from the PIV measurements, the vortex

392 center moves from the bottom left to the top right, then disappears in the street vertical

393 central plane with the increasing $R i$. Numerical simulation results from four turbulence

394 models quantitatively agree with this variation trend. When $R i \leq 0.85$ (Cases 1 and 4),

395 the agreements of SKE-SWFs with the PIV test results are better than those of RLKE-

396 SWFs and RLKE-NEWFs. When $R i>0.85$, the numerical results of RLKE model have a

397 better agreement with the PIV measurements. On the whole, it can be observed that when

$398 R i$ is small $(R i \leq 0.85)$, the SKE model can predict the flow structures better than RLKE

399 model; with the increasing $R i(R i>0.85)$, the RLKE model can perform better and better.

400 Moreover, it is important to note that when $R i$ is over 0.85 , for the same turbulence model

401 equation with the increase of $R i$ the effects of the two near-wall functions (SWFs and

402 NEWFs) on the flow structures become more and more significant. These observations

403 are generally consistent with the above-mentioned comparisons. Furthermore, it is worth

404 noting that for Case 4, there exists an obvious small vortex in the right bottom corner 
405 from both SKE and RLKE models with the SWFs near-wall treatment, however, there is

406 no obvious vortex in the right bottom corner for the wind-tunnel measurements, which

407 may indicate that due to laser light reflections on the surfaces, the flow structure very

408 close to the surfaces could not be captured easily.

409

\section{4.1.2. Temperature comparison}

411 Fluid temperature is the key parameter for the buoyancy-induced flow. Hence its

412 distribution and variation trend are worth discussing. In Fig. 10, contour plots of the

413 normalized air temperature (Eq. (21)) in the vertical central plane and the horizontal line

414 from measured data and numerical simulations are given for Cases 2, 4, 5 and 7. The

415 normalized air temperature is defined by:

$416 \quad \theta=\frac{T-T_{a}}{T_{w}-T_{a}}$

417 where $T$ represents the measured temperature $(\mathrm{K}), T_{a}$ is the ambient temperature (= $418288 \mathrm{~K})$, and $T_{w}$ is the surface temperature of the heated ground (K). Qualitatively speaking, 419 both measured and simulated results show that the high temperature region in the street 420 canyon extends from the lower-left corner to the upper-right corner, then becomes more 421 or less horizontal with the increase of Ri. Quantitatively speaking, for the following 422 situations the agreement between predicted and measured temperature contour plots in 423 the vertical central plane can be regarded quite well or acceptable: for Case $2(R i=0.1)$ 424 the predicted results by SKE-SWFs and SKE-NEWFs; for Case $4(R i=0.85)$ and Case 5 $425(R i=1.22)$ the predicted results by SKE-SWFs; and for Case $7(R i=1.56)$ the predicted 426 results by SKE-SWFs and RLKE-SWFs. Moreover, when the temperature distributions 427 of the horizontal line (line $z=0.04 \mathrm{~m}, y=0 \mathrm{~m}$ for Case 2 in Fig. 10) are concentrated, the 
428 agreement seems better and more obvious. Especially for Cases 4 and 5 the predicted

429 results by SKE-SWFs and RLKE-SWFs agree with the measured data quite well. In

430 addition, note that for the same turbulence model equation, with the increasing $R i$ the

431 effects of the two near-wall functions (SWFs and NEWFs) on the temperature

432 distributions become more and more significant. Another obvious feature is that with the

433 increase of $R i(R i \geq 0.85)$ the SKE-NEWFs and RLKE-NEWFs models overestimate the

434 temperature distributions by about $10 \% \sim 40 \%$ than wind-tunnel tests. The sudden

435 increasing temperature in the right corners of the street canyon for Cases 4 and 5 (the

436 results from PIV measurements, SKE-SWFs and RLKE-SWFs) can prove the existence

437 of the secondary vortices, although they are not captured by the PIV measurements. The

438 complicated situation of agreement between measured and numerically predicted results

439 once again show that for the mixed flow and heat transfer studied among the four

440 turbulence models no one can be regarded predominantly good. But relatively speaking,

441 for low and high $R i$ cases SKE-SWFs and RLKE-SWFs respectively behave better.

442 Therefore, in the following discussion for mass transfer only these two models are

443 adopted for simulation.

444

445 4.1.3. $S c_{t}$ effect on the pollutant distribution

446 In this section the SKE-SWFs and RLKE-SWFs turbulence models are used to

447 investigate the pollutant dispersion for Case 1 and Case 5, respectively. Pollutant

448 dispersion is a mass transfer process in nature, and the correspondent dimensionless

449 criterion is Schmidt number, which approximates the ratio of molecular momentum

450 diffusivity (kinematic viscosity) to molecular diffusivity of mass. It is worth noting that

$451 \operatorname{Pr}_{t}$ represents the ratio of turbulent momentum diffusivity and turbulent thermal 
452 diffusivity and its value of 0.85 was adopted in our simulation. In turbulence models the

453 data of turbulent Schmidt number $\left(S c_{t}\right)$ reported in the previous literatures are diverse,

454 ranging from $0.2-1.3$, basically depending on flow structures (jet, boundary layer, around

455 building, etc.) [34]. Tominaga and Stathopoulos [34] hence concluded that the value of

$456 S c_{t}$ has a significant impact on the prediction accuracy of mass transfer, and suggested the

$457 S c_{t}$ value should be selected with careful consideration of the flow characteristics. In

458 previous CFD studies for diffusion problems around building and through urban areas,

459 the values of $S c_{t}$ are quite similar (from 0.63 to 0.9) [34]. In our experiments $\mathrm{SF}_{6}$ was

460 used as the tracer gas. To the author's knowledge this tracer gas has not been used in the

461 previous studies for experimental measurement of turbulent Schmidt number. Based on

462 the above discussion, in the present simulation a series of values, $0.1,0.3,0.7$ and 1.0,

463 were used to examine its effect and try to find which one can match the test data better.

464 To ensure the comparability of the concentration distributions among the different $R i$

465 cases, the normalized concentration, $K$, is adopted defined by

$466 \quad K=c u_{h} h L / Q$

467 where $c$ is the measured mass concentration of $\mathrm{SF}_{6}\left(\mathrm{~g} / \mathrm{m}^{3}\right), u_{h}$ is the undisturbed 468 approaching flow speed $(\mathrm{m} / \mathrm{s})$ at the low-rise build height $(h=L / 2=0.8 \mathrm{~m}) ; L$ is the 469 street length $(=0.16 \mathrm{~m})$ and $Q$ is the pollutant source strength $(=0.087 \mathrm{~g} / \mathrm{s}, 0.8 \mathrm{~L} / \mathrm{min}) . \mathrm{In}$

470 the numerical solution of the governing equation of mass concentration, Eq. (6), in

471 conjunction with two turbulence models the mass wall function was assumed to have the

472 same form as for temperature, with replacing $T^{*}$ in Eq. (10) by $\theta$.

473 Figure 11 shows $K$ distributions along the vertical centerline of the street canyon from 474 the wind-tunnel tests and CFD simulations with different $S c_{t}$ values. From the 
475 comparison between the experimental and numerical results in Figs. 11(a) and (b), two

476 features can be noted. First, it can be observed that when the value of $S c_{t}$ is low the

477 concentration distribution along the height is more or less uniform, and with the increase

478 of $S c_{t}$ concentration distribution along the height becomes more and more non-uniform,

479 with the upper-center part being the largest. Second, it is noteworthy that the optimum

480 value of $S c_{t}$ revealed by the present study are both 0.7 for Cases 1 and 5 , although the

481 agreement degree of Case 5 is worse than that of Case 1. This finding shows that with

$482 \mathrm{SF}_{6}$ as the tracer gas the turbulent Schmidt number for flow and diffusion around building

483 and street canyon is quite consistent with previous studies summarized in Table 1 of

484 Tominaga and Stathopoulos [34].

\subsection{Flow-structure independence}

486 As mentioned above, the $R e$-independence concept generally is not applicable to 487 indoor flow phenomena. However, it is interesting to examine whether for the indoor 488 flow induced by flow in street canyon may exist similar phenomenon? Obviously when 489 the wind-tunnel scaled model is used to investigate the effect of street flow on the indoor 490 flow the importance of the existence of such $R e$-independence of indoor flow structure is 491 the same as the $R e$-independence of flow around building and street canyon studied by 492 wind-tunnel scaled model.

493 Figure 12(a) presents the normalized velocity distributions along the street vertical 494 centerline against the varied building Reynolds number $\left(R e_{H}\right)$ with the SKE-SWFs model. 495 Several features can be obtained. Firstly, with the increasing $R e_{H}$, the variation in the 496 velocity distributions gradually becomes mild, which implies that the difference in 497 velocity distributions is diminished with increasing $R e_{H}$. Secondly, the local diminishing 
498 rate of the different velocities varies with the height: in the local region $(0.05<z / H<0.3)$,

499 when $R e_{H}$ is over $2.16 \mathrm{E}+03\left(U_{R}>0.2 \mathrm{~m} / \mathrm{s}\right)$ the dimensionless velocities are close to each

500 other; while $z / H>0.3$ or $<0.05$, it requires $R e_{H}$ no less than $4.32 \mathrm{E}+03\left(U_{R} \geq 0.4 \mathrm{~m} / \mathrm{s}\right)$ to

501 ensure the normalized velocity distributions squeeze into a narrow region. From the

502 above observation, it can be concluded that for the external flow in the street scale, there

503 exists a critical $R e_{H}\left(R e_{H, c r i t}\right)$, over which the flow structure can be regarded as

504 independence on the $R e_{H}$, for which the suggested value is equal to $4.32 \mathrm{E}+03$.

505 Figure 12(b) shows the numerically predicted dimensionless velocity contours and

506 streamlines in the room vertical central plane with different $R e_{H}$. It can be observed that 507 the flow structures in the room change a lot with the increasing of $R e_{H}$ when the value of

$508 R e_{H}$ is less than 7.57E+03, however, when the value of $R e_{H}$ is larger than 7.57E+03 $\left(U_{R} \geq\right.$

$5090.7 \mathrm{~m} / \mathrm{s}$ ) not too much effect of $R e_{H}$ on the indoor flow structure can be found, and the

510 coordinates of the vortex center in the indoor vertical central plane almost keeps

511 unchanged. Thus for the situation studied $7.57 \mathrm{E}+03$ can be regarded as the critical

512 Reynolds number for the $R e$-independence of indoor flow structure, which is appreciably

513 larger than $R e_{H, c r i t}$ mentioned above (4.32E+03). This is perhaps because the indoor flow

514 is affected by the relatively stronger viscous force generated by the surrounding walls. To

515 make it meaningful for the wind-tunnel tests in both street and indoor scales, we suggest

516 the value of $R e_{H, c r i t}$ for this multiscale model is $7.57 \mathrm{E}+03$, which indicates that the

517 smallest velocity for the wind-tunnel tests is $U_{R}=0.7 \mathrm{~m} / \mathrm{s}$ for this study.

$518 \quad 4.3$ The effects of outdoor environment on the IAQ

519 The physical phenomenon of the effects of outdoor environment on the IAQ can be 520 described as follows: the pollutants released from the stack on the rooftop of the low-rise 
521 building follow with the mixed flow stream coming from the neighborhood scale into the

522 street canyon (street scale), then enter into the indoor scale of the high-rise building

523 through the ventilation and affect the health of residents in the rooms. So the IAQ is

524 mainly determined by the environmental conditions near the open windows in the street

525 scale. For the problem studied in this paper the environmental conditions in the street

526 scale are dependent on both inertial force and buoyancy force.

527 To investigate this effect, the normalized pollutant distributions $(K)$ along the room

528 horizontal centerline for the eight studied cases (in Table 1) are determined from wind-

529 tunnel test and compared with CFD simulations (Cases 1-4 using SKE-SWFs; Cases 5-6

530 using RLKE-SWFs; Cases 7-8 using RLKE-NEWFs) in Fig. 13, where the same shape of

531 symbols are adopted for both measured (larger one) and simulated results (small one).

532 From the analysis of flow-structure independence in Section 4.2, the wind-tunnel results

533 from Cases 1-7 can represent the realistic cases, although there exist some errors in Case

534 8. In addition, the turbulent models and near-wall treatments are chosen because they

535 perform better for predicting the flow fields in the street and indoor scale models. An

536 obvious feature of Fig. 13 is the almost uniform values of the normalized pollutant

537 distributions for each case, which can be attributed to the good mixing for the airflow in

538 the rooms. Secondly, it can be seen that the numerical models applied can perform well to

539 predict the pollutant distributions in the indoor scales for the eight studied cases. Thirdly,

540 it is noted that for Cases 5 and 6 (the same street $R i$ ) both the experimental and numerical

541 results agree with each other quite well, especially the predicted results of the two cases

542 are totally coincide, which indicates that the mixed pollutant distributions in the indoor

543 scale are mainly dependent on the street $R i$. Furthermore, it is worth nothing that with the 
544 increasing $R i$ the average pollutant concentrations along the horizontal centerlines in the

545 indoor scale models first increase from Case 1 to Case 4, then decrease from Case 4 to

546 Case 8. Therefore, in this studied cases there exists the most disadvantage case (Case 4,

$547 R i=0.85)$ to cause the worst IAQ. The above physical phenomenon can be explained as

548 follows: with the increase of $R i$ from 0 to 0.85 (Case 1 to Case 4), the vortex centers in the

549 street vertical plane approach the street center gradually [24] to cause the air flow with

550 higher pollutant concentrations nearby the open window (see Cases 1 and 4 in Fig.7).

551 However, with the continuous increase of $R i$ ( $R i$ over 1.22, Case 5), the buoyancy force

552 seems more obvious and strong enough to push the whole vortex structure to move

553 towards the top-right of the street vertical plane until the vortex disappears (see Cases 5

554 and 7 in Fig. 7). Meanwhile, with the increasing $R i$, more and more clean air coming

555 from the both sides of the street canyon is carried into the vortex structures in the street

556 canyon, and dilutes the pollutant concentrations there, which is one of the main reason to

557 cause the $K$ vs. $R i$ variation in the indoor scale models.

\section{5. Conclusion}

559 In this study, a multiscale problem on the air flow and pollutant dispersion passing 560 over the neighborhood scale - street scale - indoor scale models was studied by the 561 coupled CFD models and the wind-tunnel measurements with a wide range of $R i$ from 0 562 to 4.77. Two different turbulence models (SKE and RLKE) with two near-wall treatment

563 methods (SWFs and NEWFs) were applied in the coupled CFD models, then numerical

564 models were used to investigate the multiscale problem further. The following 565 conclusions can be obtained.

566 (1) The adopted coupled CFD models can predict the general flow structures and the 
567 buoyancy effects. When $R i \leq 0.85$, the standard $k-\varepsilon$ model (SKE) can better predict the 568 flow fields and temperature distributions. When $R i>0.85$, the realizable $k-\varepsilon$ model 569 (RLKE) can perform better. For the same turbulence model equation with the increase of

570 Ri the effects of the two near-wall functions (SWFs and NEWFs) on the flow structures

571 and temperature distributions become more and more significant.

572 (2) When $\mathrm{SF}_{6}$ is used as the tracer gas the optimal value of $S c_{t}$ is 0.7 for the studied 573 multiscale models.

574 (3) It is numerically found that for the indoor flow structure caused by an outer street 575 flow there also exists a $R e$-independence region.

576 (4) For the related indoor flow of the multiscale model studied, the critical Reynolds

577 number based on build height is $7.57 \mathrm{E}+03$ and only when the street $R e_{H}$ is larger than this

578 value, the wind-tunnel results both in the street and indoor scale models are meaningful 579 to represent the realities.

580 (5) The IAQ (indoor scale) is mainly affected by the outdoor environments (street scale) 581 through the open windows (ventilation). For the multiscale model studied with the 582 increase of $R i$ the average pollutant concentrations in the indoor scale first increase and 583 then decrease.

585 Acknowledgments

586 This work is supported by the National Science Foundation of China (Grant No. 587 51136004), the $12^{\text {th }}$ Five-Year National Key Technology R\&D Program 588 (2012BAJ02B03), and the Fundamental Research Funds for the Central Universities. 589 Wind-tunnel test was conducted in the TJ-1 and TJ-4 wind tunnel of the State Key 590 Laboratory of Civil Engineering for Disaster Prevention, Tongji University. 


\section{References}

593 [1] E. Wennan, B. Engquist, Multiscale modeling and computation, Not. Am. Math. Soc., 50 (9) (2003)

$5941062-1070$.

595 [2] Y.L. He, W.Q. Tao, Multiscale simulations of heat transfer and fluid flow problems. J. heat transfer 134

$596 \quad$ (3) (2012) 031018.

597 [3] J.C. Chow, J.G. Watson, P.K.K. Louie, L.W.A. Chen, D. Sin, Comparison of PM2.5 carbon 598 measurement methods in Hong Kong, China. Environ. Pollut. 137 (2) (2005) 334 - 344.

599 [4] Z. Niu, S. Wang, J. Chen, F.W. Zhang, X.Q. Chen, C. He, L.F. Lin, L.Q. Yin, L.L. Xu, Source 600 contributions to carbonaceous species in PM 2.5 and their uncertainty analysis at typical urban, peri-urban 601 and background sites in southeast China. Environ. Pollut. 181 (2013) 107-114.

602 [5] Y.J. Liu, T.T. Zhang, Q.Y. Liu, R.J. Zhang, Z.Q. Sun, M.G. Zhang, Seasonal variation of physical and 603 chemical properties in TSP, PM10 and PM2.5 at a roadside site in Beijing and their influence on 604 atmospheric visibility. Aerosol. Air Qual. Res. 14 (3) (2014) 954 - 969.

605 [6] L.J. Han, W.Q. Zhou, W.F. Li, L. Li, Impact of urbanization level on urban air quality: A case of fine 606 particles (PM2.5) in Chinese cities. Environ. Pollut. 194 (2014) 163 - 170.

607 [7] R.L. Buckley, C.H. Hunter, D.W. Werth, M.T. Whiteside, K.F. Chen, C.A. Mazzola, A case study of 608 chlorine transport and fate following a large accidental release. Atmos. Environ. 62 (2012) $184-198$.

609 [8] M.F. Milazzo, G. Ancione, R. Lisi, C. Vianello, G. Maschio, Risk management of terrorist attacks in the 610 transport of hazardous materials using dynamic geoevents. J. Loss Prevent. Proc. 22 (5) (2009) 625 - 633.

611 [9] R.E. Britter, S.R. Hanna, Flow and dispersion in urban areas. Annu. Rev. Fluid Mech. 35 (1) (2013) $612469-496$.

613 [10] T. van Hooff, B. Blocken, Full-scale measurements of indoor environmental conditions and natural 614 ventilation in a large semi-enclosed stadium: Possibilities and limitations for CFD validation. J. Wind Eng. 615 Ind. Aerod. 104 (2012) 330 - 341.

616 [11] P. Gousseau, T. Blocken, T. Stathopoulos, G.J.F. van Heijst, CFD simulation of near-field pollutant 617 dispersion on a high-resolution grid: A case study by LES and RANS for a building group in downtown 618 Montreal. Atmos. Environ. 45 (2011) 428 - 438.

619 [12] J. Allegrini, V. Dorer, J. Carmeliet, Buoyant flows in street canyons: Validation of CFD 620 simulations with wind tunnel measurements. Build. Environ. 72 (2014) 63 - 74.

621 [13] G. M. Stavrakakis, M. K. Koukou, M. G. Vrachopoulos, N. C. Markatos, Natural cross-ventilation in 622 buildings: Building-scale experiments, numerical simulation and thermal comfort evaluation. Energy Build. $62340(9)(2008) 1666-1681$. 
624 [14] T. van Hooff, B. Blocken, Coupled urban wind flow and indoor natural ventilation modelling on a

625 high-resolution grid: A case study for the Amsterdam ArenA stadium. Environ. Model. Soft. 25 (1) (2010) $62651-65$.

627 [15] Y. Jiang, Q. Chen, Effect of fluctuating wind direction on cross natural ventilation in buildings from 628 large eddy simulation. Build. Environ. 37 (4) (2002) 379 - 386.

629 [16] M.J. Cook, Y. Ji, G.R. Hunt, CFD modeling of natural ventilation: combined wind and buoyancy 630 forces. Int. J. Vent. 1 (3) (2003) $169-180$.

631 [17] Q. Chen, Ventilation performance prediction for buildings: a method overview and recent applications.

632 Build. Environ. 44 (4) (2009) 848 - 858.

633 [18] Q.H. Nie, Y. Joshi, Multiscale thermal modeling methodology for thermoelectrically cooled electronic 634 cabinet, Numer. Heat Trans. A-Appl. 53 (3) (2008) 225 - 248.

635 [19] P.Y. Cui, Z. Li, W.Q. Tao, Wind tunnel experiments and multiscale modeling for effects of traffic 636 exhausts on the indoor air quality within urban-scale regions. Indoor Air 2014 - 13th International 637 Conference on Indoor Air Quality and Climate, (2014) 545 - 552.

638 [20] T. van Hooff, B. Blocken. On the effect of wind direction and urban surroundings on natural 639 ventilation of a large semi-enclosed stadium. Comput. Fluids, 39 (7) (2010) 1146 - 1155.

640 [21] P.Y. Cui, Z. Li, W.Q. Tao, Investigation of Re-independence of turbulent flow and pollutant 641 dispersion in urban street canyon using numerical wind tunnel (NWT) models. Int. J. Heat Mass Transfer 64279 (2014) $176-188$.

643 [22] W.H. Snyder, Similarity criteria for the application of fluid models to the study of air pollution 644 meteorology. Boundary-Layer Meteorol. 3 (1972) 113 - 134.

645 [23] K. Uehara, S. Murakami, S. Oikawa, S. Wakamatsu, Wind tunnel experiments on how thermal 646 stratification affects flow in and above urban street canyons. Atmos. Environ. 34 (2000) 1553 - 1562.

647 [24] P.Y. Cui, Z. Li, W.Q. Tao, Wind-tunel measurements for the thermal effects on the air flow and 648 pollutant dispersion through different scale urban areas. Build. Environ. 97 (2016) 137 - 151.

649 [25] W.Q. Tao, Numerical Heat Transfer, second ed., Xi'an Jiaotong University Press, Xi'an, 2001.

650 [26] B.E. Launder, D.B. Spalding, The numerical computation of turbulent flows. Comput. Methods Appl. 651 Mech. Eng. 3 (2) (1974) $269-289$.

652 [27] C. Jayatillaka, The influence of prandtl number and surface roughness on the resistance of the laminar 653 sublayer to momentum and heat transfer. Prog. Heat Mass Transfer 1 (1969) 193 - 321.

654 [28]S.E. Kim, D. Choudhury, A near-wall treatment using wall functions sensitized to pressure gradient. In: 655 ASME FED (Ed.), Symp. on Separated and Complex Flows 217 (1995) 249 - 256.

656 [29] J. Franke, A. Hellsten, H. Schlünzen, B. Carissimo, Best practice guideline for the CFD simulation of 657 flows in the urban environment, 2007 May.

658 [30] S.V. Patankar, Numerical Heat Transfer and Fluid Flow, CRC Press, New York, 1980.

659 [31] Fluent Inc.,2006. Fluent user's guide. 
660 [32] J. Franke, A. Hellsten, K.H. Schlunzen, B. Carissimo, The cost 732 best practice guideline for CFD

661 simulation of flows in the urban environment: a summary. Int. J. Environ. Pollut. 44 (1-4) (2011) 419 662427.

663 [33] S. Di Sabatino, R. Buccolieri, B. Pulvirenti, R. Britter, Simulations of pollutant dispersion within 664 idealised urban-type geometries with CFD and integral models. Atmos. Environ. 41 (37) (2007), 8316 6658329.

666 [34] Y. Tominaga, T. Stathopoulos, Turbulent Schmidt numbers for CFD analysis with various types of 667 flowfield. Atmos. Environ. 41 (37) (2007) 8091 - 8099. 


\section{Captions}

Table 1 Parameters for the eight studied cases.

Table 2 The governing equations for two $k-\varepsilon$ turbulence models.

Table 3 The boundary conditions of the CFD simulations. 
Table 1 Parameters for the eight studied cases.

\begin{tabular}{cccccccc}
\hline Cases & $U_{R}(\mathrm{~m} / \mathrm{s})$ & $\Delta T\left({ }^{\circ} \mathrm{C}\right)$ & $G r$ & $R e$ & $R i=G r / R e^{2}$ & $\beta \cdot \Delta T$ & Density $(\rho)$ \\
\hline 1 & 6.0 & 0 & 0 & $6.49 \mathrm{E}+04$ & 0 & 0 & Constant \\
\hline 2 & 2.8 & 13 & $8.10 \mathrm{E}+06$ & $3.03 \mathrm{E}+04$ & 0.01 & 0.04 & Boussinesq \\
3 & 1.4 & 55.5 & $3.23 \mathrm{E}+07$ & $1.51 \mathrm{E}+04$ & 0.14 & 0.18 & approximation \\
\hline 4 & 0.7 & 88.5 & $4.89 \mathrm{E}+07$ & $7.57 \mathrm{E}+03$ & 0.85 & 0.27 & \\
5 & 0.7 & 135 & $6.97 \mathrm{E}+07$ & $7.57 \mathrm{E}+03$ & 1.22 & 0.38 & Incompressible \\
6 & 0.8 & 185 & $8.93 \mathrm{E}+07$ & $8.56 \mathrm{E}+03$ & 1.22 & 0.49 & ideal gas law \\
7 & 0.7 & 185 & $8.93 \mathrm{E}+07$ & $7.57 \mathrm{E}+03$ & 1.56 & 0.49 & \\
8 & 0.4 & 185 & $8.93 \mathrm{E}+07$ & $4.32 \mathrm{E}+03$ & 4.77 & 0.49 & \\
\hline
\end{tabular}


Table 2 The governing equations for two $k-\varepsilon$ turbulence models.

\begin{tabular}{|c|c|c|c|}
\hline & Eddy viscosity $\eta_{t}$ & Transport equation of $k$ & Transport equation of $\varepsilon$ \\
\hline $\begin{array}{l}\text { Standard } \\
k-\varepsilon \text { model } \\
(\mathrm{SKE})\end{array}$ & $\begin{array}{l}\eta_{t}=\rho C_{\mu} \frac{k^{2}}{\varepsilon} \\
C_{\mu}=0.09\end{array}$ & $\begin{array}{l}\frac{\partial(\rho k)}{\partial t}+\frac{\partial\left(\rho k u_{j}\right)}{\partial x_{j}}=\frac{\partial}{\partial x_{j}}\left[\left(\eta+\frac{\eta_{t}}{\sigma_{k}}\right) \frac{\partial k}{\partial x_{j}}\right]+ \\
G_{k}-\rho \varepsilon \\
G_{k}=\eta_{t} \frac{\partial u_{i}}{\partial x_{j}}\left(\frac{\partial u_{i}}{\partial x_{j}}+\frac{\partial u_{j}}{\partial x_{i}}\right) \quad \sigma_{k}=1.0\end{array}$ & $\begin{array}{l}\frac{\partial(\rho \varepsilon)}{\partial t}+\frac{\partial\left(\rho \varepsilon u_{j}\right)}{\partial x_{j}}=\frac{\partial}{\partial x_{j}}\left(\left(\eta+\frac{\eta_{t}}{\sigma_{\varepsilon}}\right) \frac{\partial \varepsilon}{\partial x_{j}}\right)+ \\
\frac{\varepsilon}{k}\left(C_{\varepsilon 1} G_{k}-C_{\varepsilon 2} \rho \varepsilon\right) \\
C_{\varepsilon 1}=1.44, C_{\varepsilon 2}=1.92, \sigma_{\varepsilon}=1.3\end{array}$ \\
\hline $\begin{array}{c}\text { Realizabl } \\
\text { e } k-\varepsilon \\
\text { model } \\
\text { (RLKE) }\end{array}$ & $\begin{array}{l}\eta_{t}=\rho C_{\mu} \frac{k^{2}}{\varepsilon}, C_{\mu}=\frac{1}{4.04+A_{S} k u^{*} / \varepsilon} \\
u^{*}=\sqrt{S_{i j} S_{i j} \tilde{\Omega}_{i j} \widetilde{\Omega}_{i j}, \widetilde{\Omega}_{i j}=\Omega_{i j}-2 \varepsilon_{i j k} \omega_{k}} \\
A_{S}=\sqrt{6} \cos \phi, \phi=\frac{1}{3} \arccos (\sqrt{6} W) \\
W=\frac{S_{i j} S_{i k} S_{j k}}{\tilde{S}^{3}}, \tilde{S}=\sqrt{S_{i j} S_{i j}}\end{array}$ & $\begin{array}{l}\frac{\partial(\rho k)}{\partial t}+\frac{\partial\left(\rho k u_{j}\right)}{\partial x_{j}}=\frac{\partial}{\partial x_{j}}\left(\left(\eta+\frac{\eta_{t}}{\sigma_{k}}\right) \frac{\partial k}{\partial x_{j}}\right)+ \\
G_{k}-\varepsilon, \quad \sigma_{k}=1.0\end{array}$ & $\begin{array}{l}\frac{\partial(\rho \varepsilon)}{\partial t}+\frac{\partial\left(\rho \varepsilon u_{j}\right)}{\partial x_{j}}=\frac{\partial}{\partial x_{j}}\left(\left(\eta+\frac{\eta_{t}}{\sigma_{\varepsilon}}\right) \frac{\partial \varepsilon}{\partial x_{j}}\right)+ \\
C_{1} \rho S_{\varepsilon}-C_{2} \rho \frac{\varepsilon^{2}}{k+\sqrt{v \varepsilon}} \\
C_{1}=\max \left[0.43, \frac{\tilde{\eta}}{\tilde{\eta}+5}\right], C_{2}=1.9, \sigma_{\varepsilon}=1.2\end{array}$ \\
\hline
\end{tabular}


Table 3 The boundary conditions of the CFD simulations.

\begin{tabular}{cccc}
\hline Boundary conditions & Velocity & Temperature & Pollutant \\
\hline Inlet plane & Constant & $T_{a}$ & 0 \\
Outlet plane & $d u / d x=0$ & $d T / d x=0$ & $d c / d x=0$ \\
& $d u / d y=0 ;$ & & \\
Center plane & $d w / d y=0 ; v=0$. & $d T / d y=0$ & $d c / d y=0$ \\
& $d u / d y=0 ;$ & & \\
Top/right/left planes & $d w / d y=0 ; v=0$. & $d T / d y=0$ & $d c / d y=0$ \\
Bottom plane & $\mathrm{SWFs} \mathrm{or} \mathrm{NEWFs}$ & $T_{a}$ & 0 \\
Street ground & $\mathrm{SWFs}$ or NEWFs & $T$ & 0 \\
Pollutant source & 0 & $T_{a}$ & Constant \\
\hline
\end{tabular}




\section{Captions}

Fig. 1. Multiscale phenomena of air flow and pollutant dispersion in urban areas.

Fig. 2. The configurations of multiscale models and experimental setup.

Fig. 3. Computational domain and grid generation.

Fig. 4. The grid independence analysis (velocity distribution along the horizontal centerline of the room).

Fig. 5. Normalized velocity distributions along the vertical centerline of the street canyon for different $R i$ cases. Wind tunnel measurements and CFD simulations by using four turbulence models (SKE-SWFs, SKE-NEWFs, RLKE-SWFs and RLKE-NEWFs).

Fig. 6. Correlation coefficients $\left(R^{2}\right)$ between wind tunnel measurements and different numerical models along the vertical center line in the street scale.

Fig. 7. Normalized velocity distributions along the horizontal centerline in the indoor scale for different $R i$ cases. Wind tunnel measurements and CFD simulations by using four turbulence models (SKE-SWFs, SKE-NEWFs, RLKE-SWFs and RLKE-NEWFs).

Fig. 8. Correlation coefficients $\left(R^{2}\right)$ among different numerical models compared between the street scale and indoor scale.

Fig. 9. Normalized streamlines in the vertical central plane of street canyon. Wind tunnel measurements and CFD simulations by using four turbulence models (SKE-SWFs, SKE-NEWFs, RLKE-SWFs and RLKE-NEWFs).

Fig. 10. Distributions of dimensionless temperature $(\theta)$ in the vertical central plane of street canyon for different cases. Wind tunnel measurements and CFD simulations by using four turbulence models (SKE-SWFs, SKE-NEWFs, RLKE-SWFs and RLKE-NEWFs).

Fig. 11. Normalized concentration distributions along the vertical centerline of the street canyon. Wind tunnel measurements and CFD simulations by using two turbulence models (SKE-NEWFs and RLKE-SWFs) with different $S c_{t}$ numbers.

Fig. 12. Flow structure independence analysis for the street scale and indoor scale models.

Fig. 13. Normalized pollutant distributions along the room horizontal center lines for different cases. Wind tunnel measurements (large symbols) and CFD simulations (small symbols). 


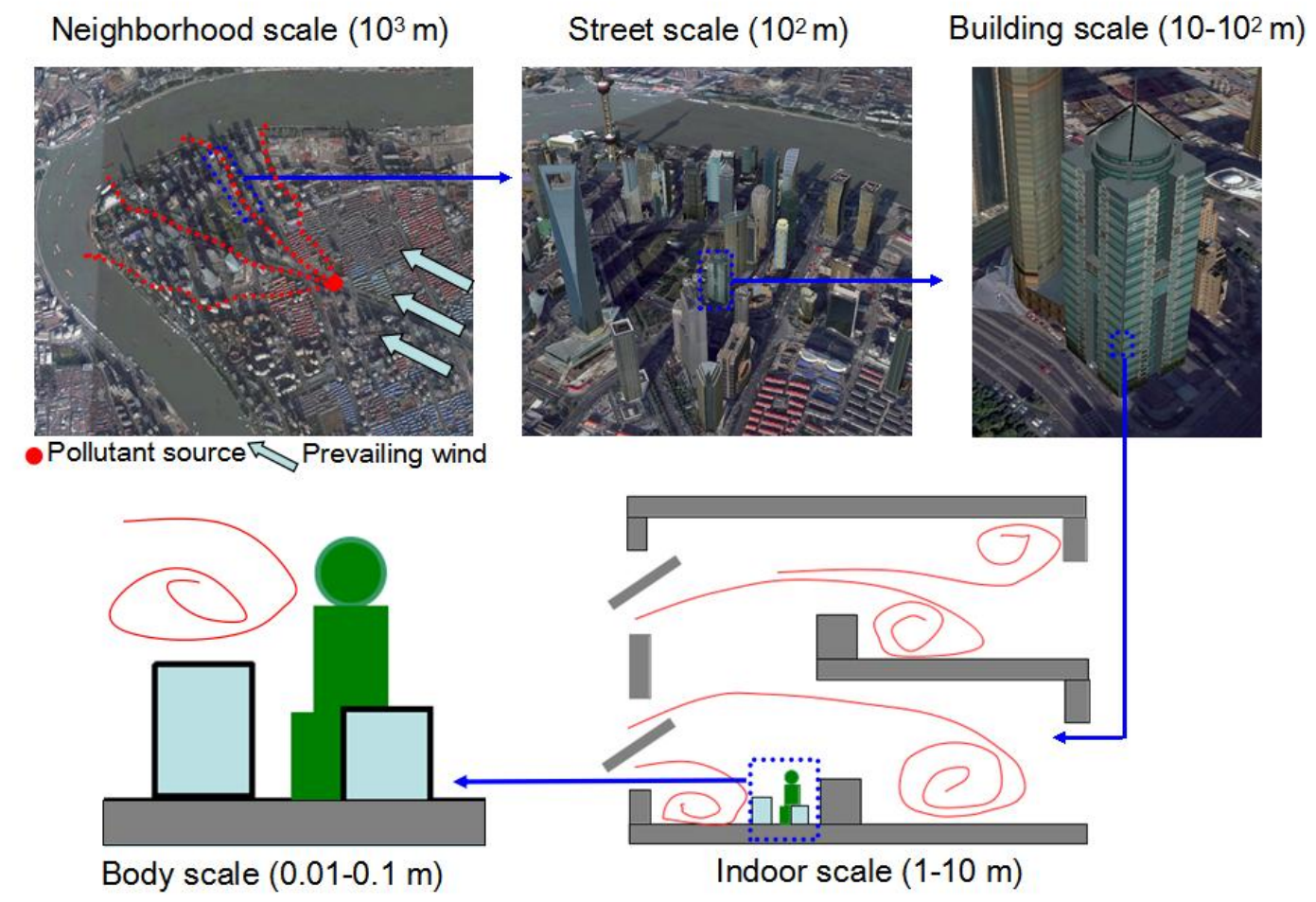

Fig. 1. Multiscale phenomena of air flow and pollutant dispersion in urban areas. 


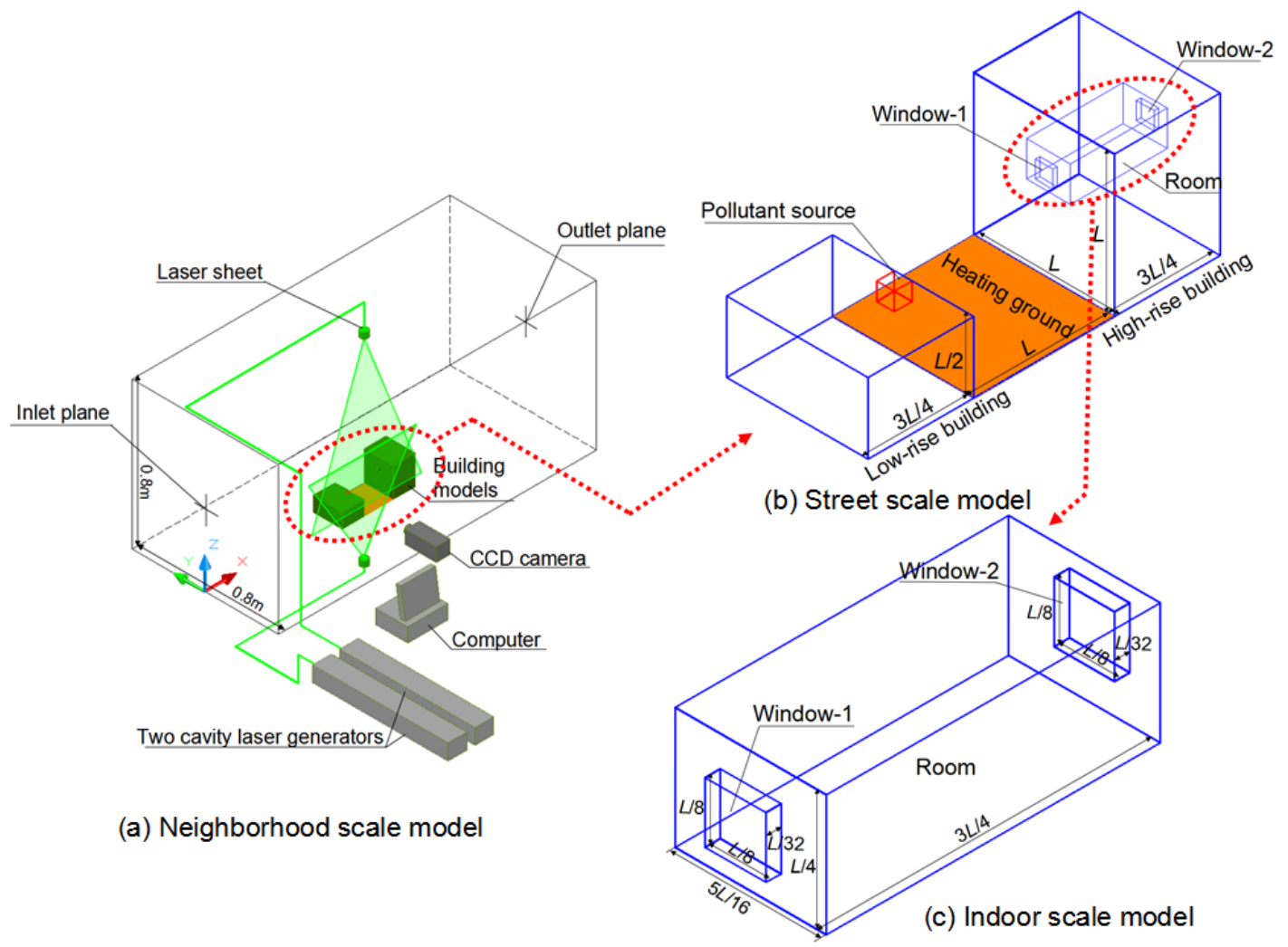

Fig. 2. The configurations of multiscale models and experimental setup. 


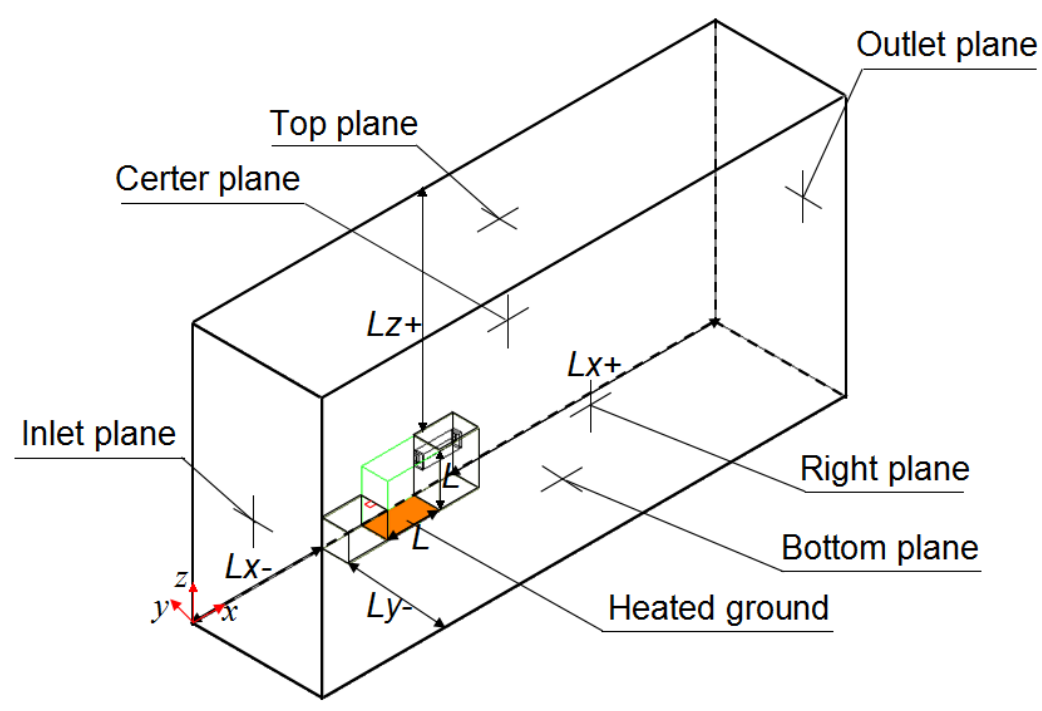

(a) Computational domain

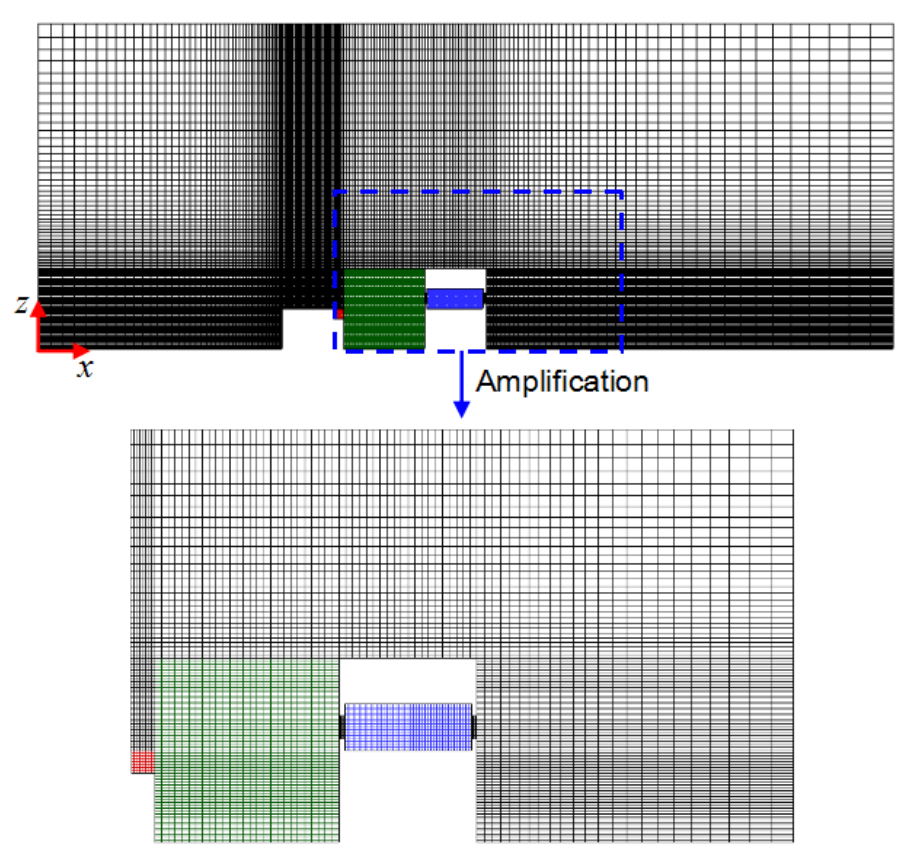

(b) Grid display on $x-z$ plane

Fig. 3. Computational domain and grid generation. 


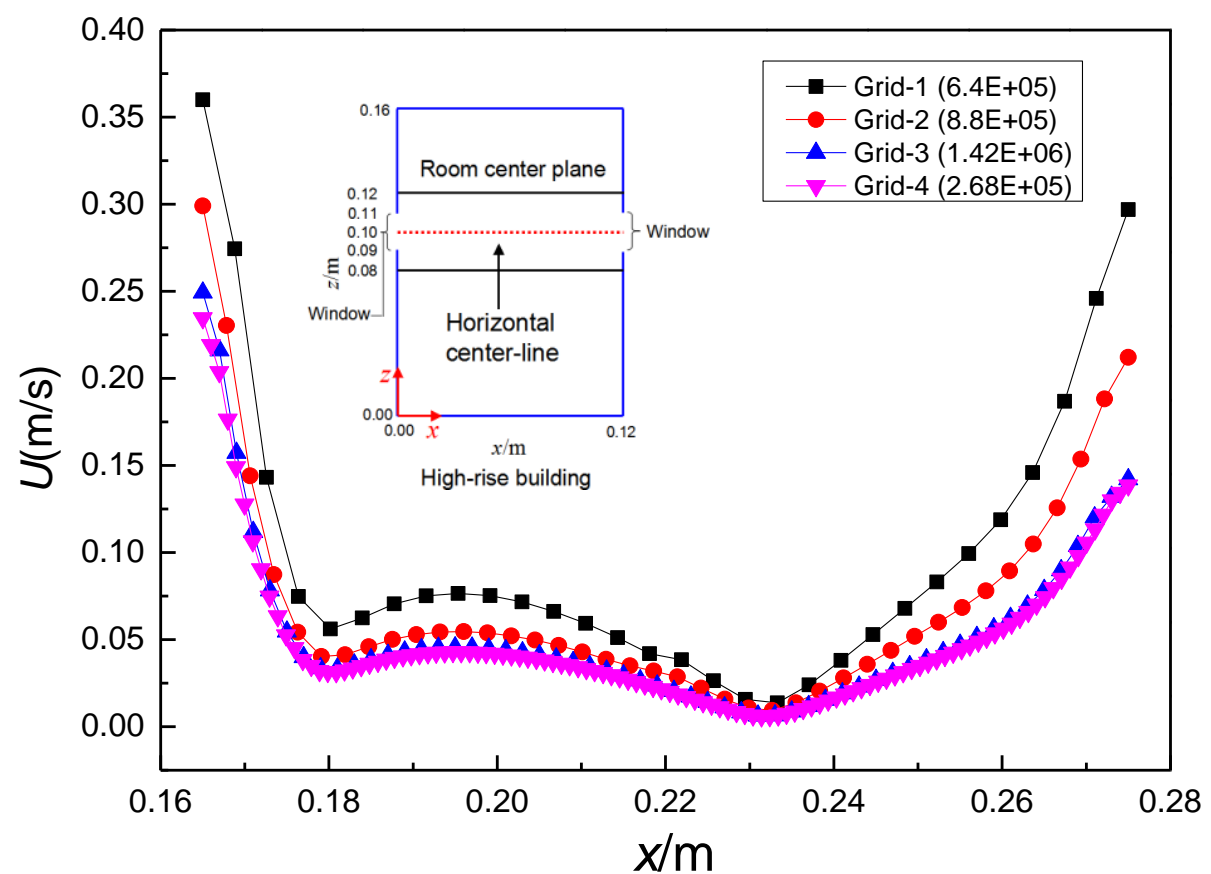

Fig. 4. The grid independence analysis (velocity distribution along the horizontal centerline of the room). 


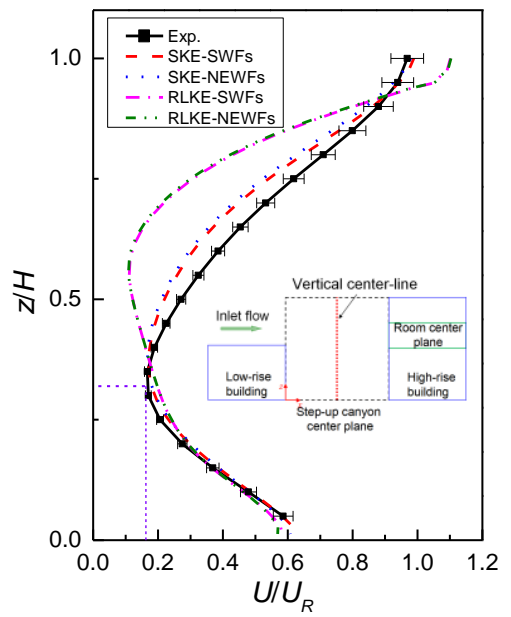

Case 1: $R i=0, R e=6.49 \mathrm{E}+04, G r=0$

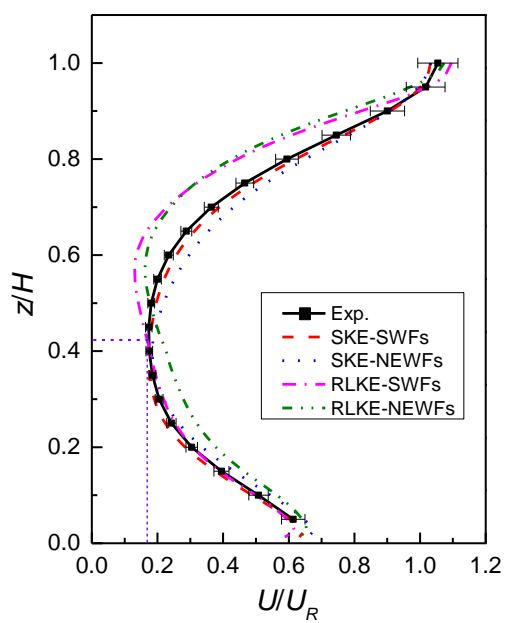

Case 3: $R i=0.14, R e=1.51 \mathrm{E}+04, G r=3.23 \mathrm{E}+07$

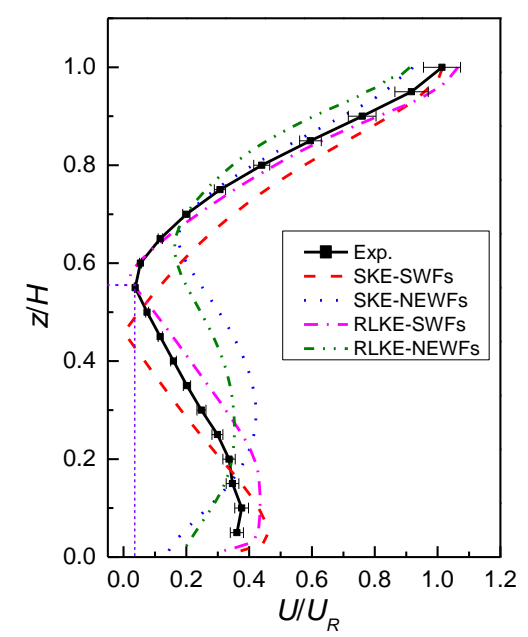

Case 5: $R i=1.22, R e=7.57 \mathrm{E}+03, G r=6.97 \mathrm{E}+07$

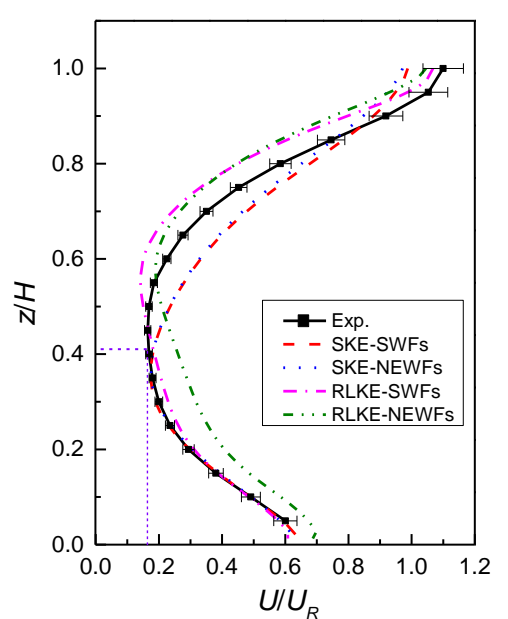

Case 2: $R i=0.01, R e=3.03 \mathrm{E}+04, G r=8.10 \mathrm{E}+06$

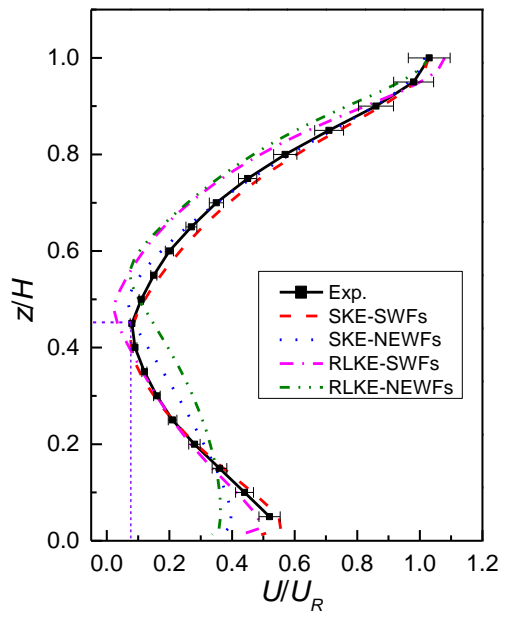

Case 4: $R i=0.85, R e=7.57 \mathrm{E}+03, G r=4.89 \mathrm{E}+07$

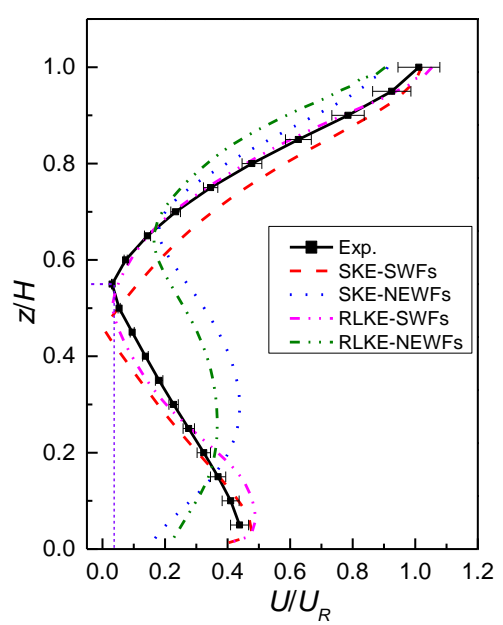

Case 6: $R i=1.22, R e=8.56 \mathrm{E}+03, G r=8.93 \mathrm{E}+07$ 


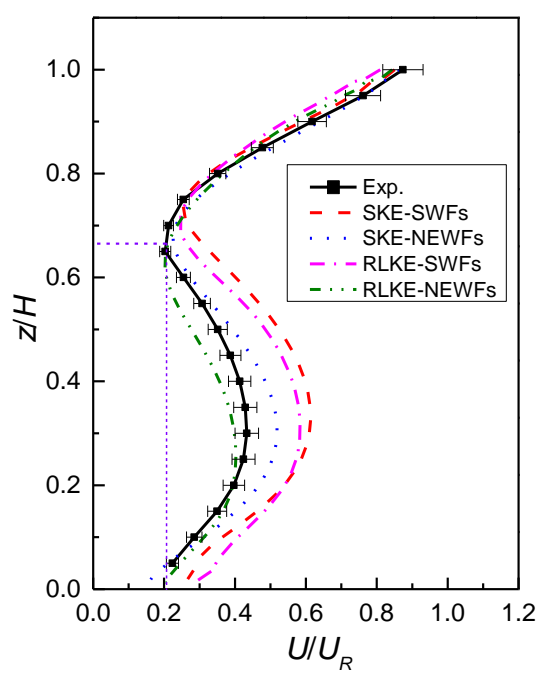

Case 7: $R i=1.56, R e=7.57 \mathrm{E}+03, G r=8.93 \mathrm{E}+07$

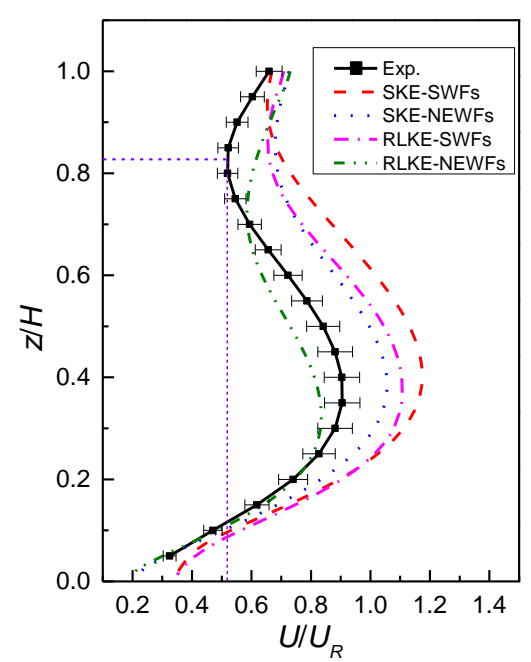

Case 8: $R i=4.77, R e=4.32 \mathrm{E}+03, G r=8.93 \mathrm{E}+07$

Fig. 5. Normalized velocity distributions along the vertical centerline of the street canyon for different $R i$ cases. Wind tunnel measurements and CFD simulations by using four turbulence models (SKE-SWFs, SKE-NEWFs, RLKE-SWFs and RLKE-NEWFs). 


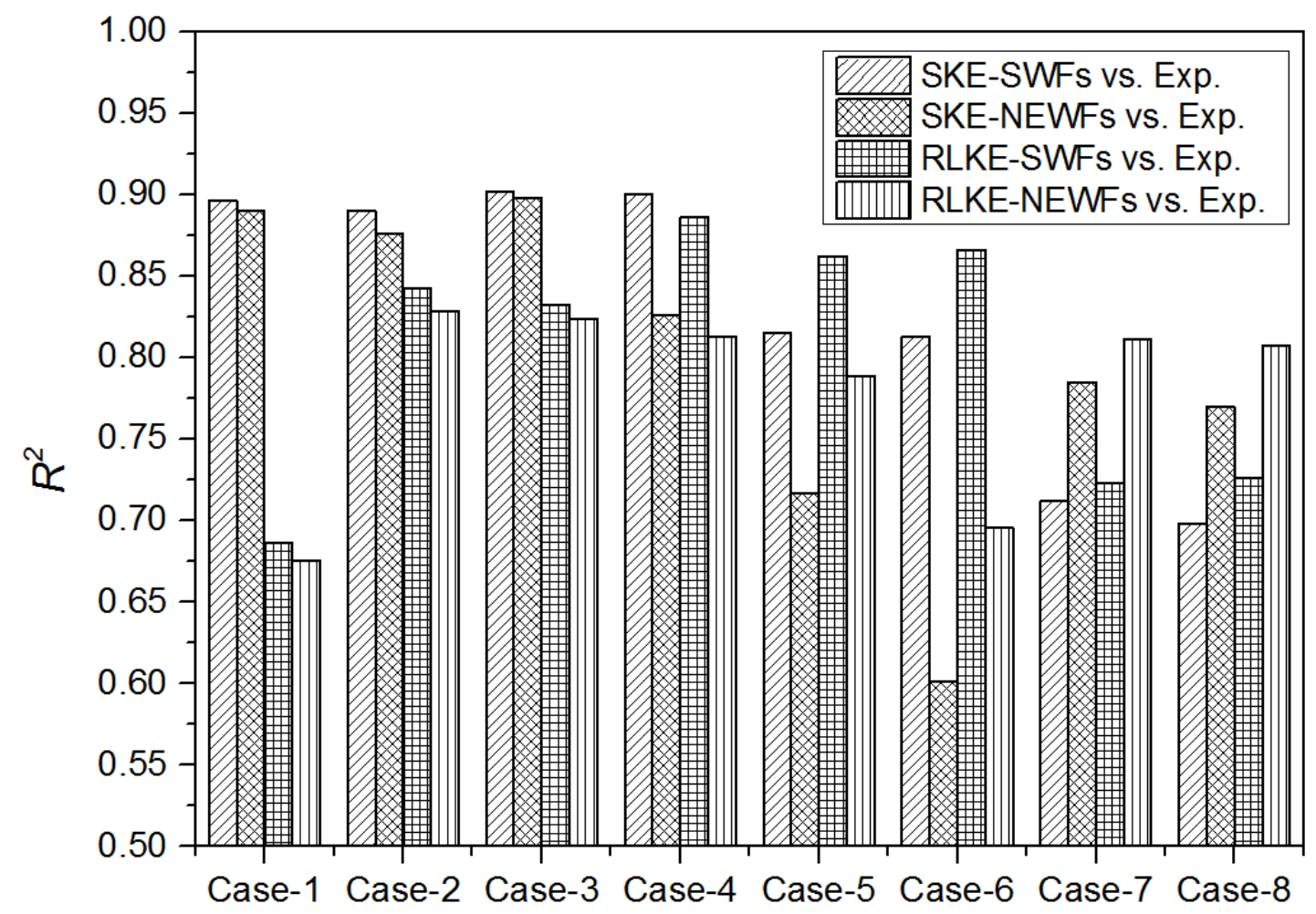

Fig. 6. Correlation coefficients $\left(R^{2}\right)$ between wind tunnel measurements and different numerical models along the vertical centerline in the street scale. 


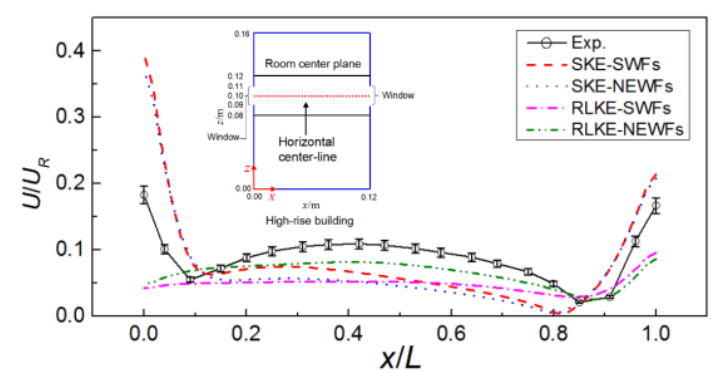

Case 1: $R i=0, R e=6.49 \mathrm{E}+04, G r=0$

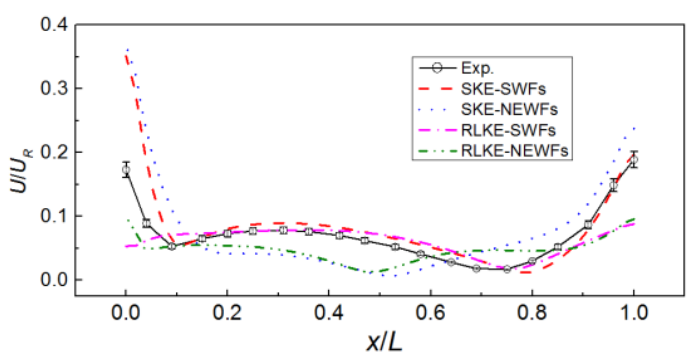

Case 3: $R i=0.14, R e=1.51 \mathrm{E}+04, G r=3.23 \mathrm{E}+07$

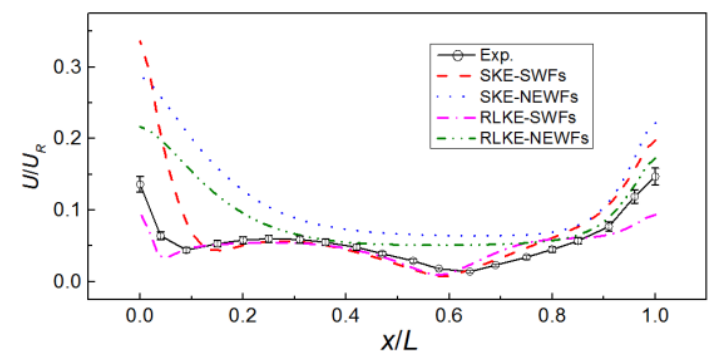

Case 5: $R i=1.22, R e=7.57 \mathrm{E}+03, G r=6.97 \mathrm{E}+07$

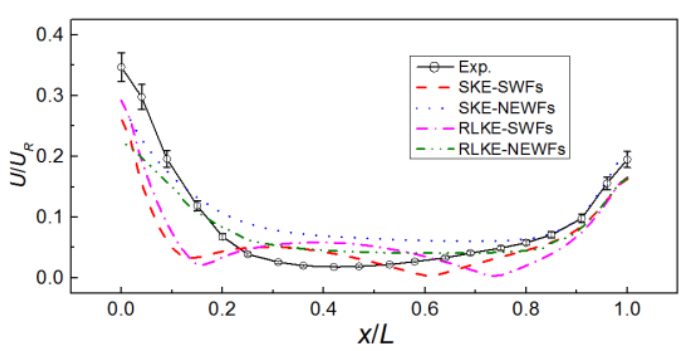

Case 7: $R i=1.56, R e=7.57 \mathrm{E}+03, G r=8.93 \mathrm{E}+07$

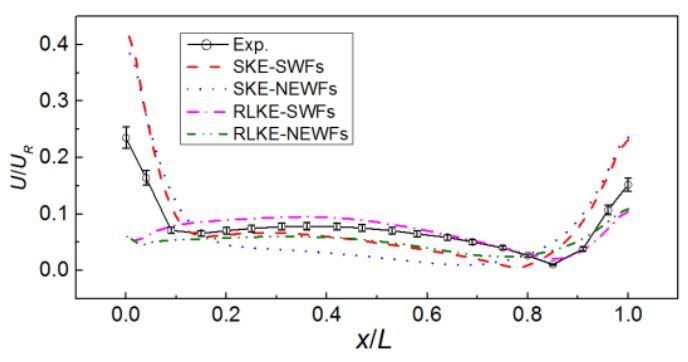

Case 2: $R i=0.01, R e=3.03 \mathrm{E}+04, G r=8.10 \mathrm{E}+06$

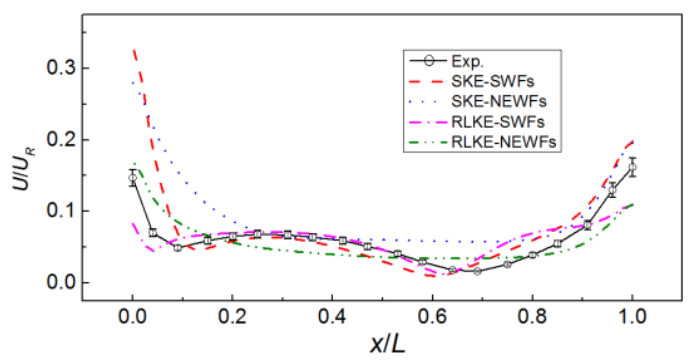

Case 4: $R i=0.85, R e=7.57 \mathrm{E}+03, G r=4.89 \mathrm{E}+07$

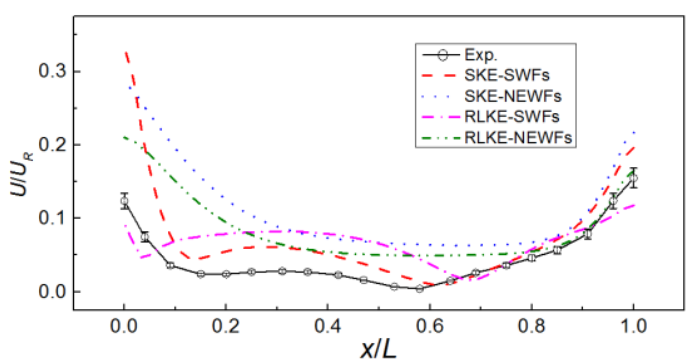

Case 6: $R i=1.22, R e=8.56 \mathrm{E}+03, G r=8.93 \mathrm{E}+07$

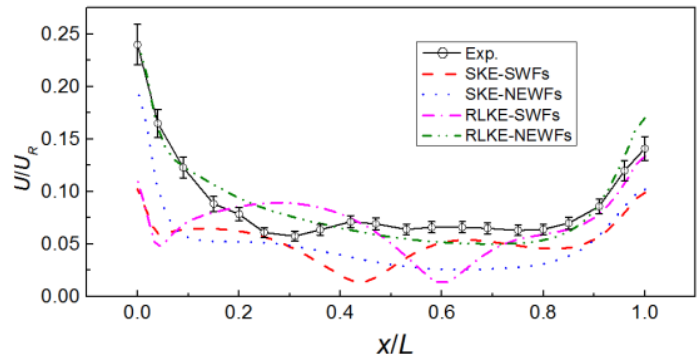

Case 8: $R i=4.77, R e=4.32 \mathrm{E}+03, G r=8.93 \mathrm{E}+07$

Fig. 7. Normalized velocity distributions along the horizontal center line in the indoor scale for different $R i$ cases. Wind tunnel measurements and CFD simulations by using four turbulence models (SKE-SWFs, SKE-NEWFs, RLKE-SWFs and RLKE-NEWFs). 


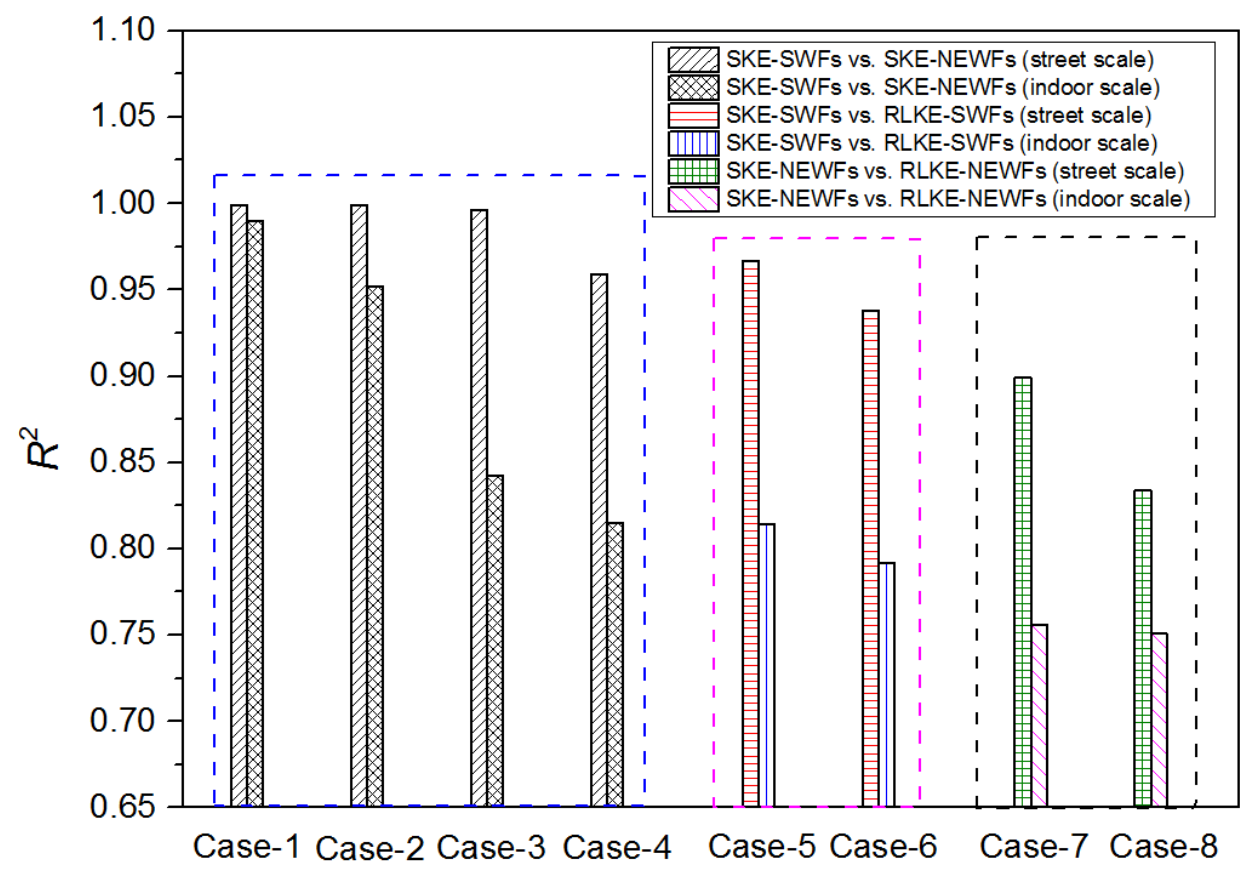

Fig. 8. Correlation coefficients $\left(R^{2}\right)$ among different numerical models compared between the street scale and indoor scale. 


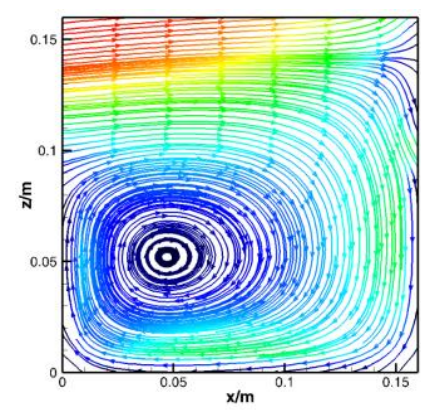

PIV measurement

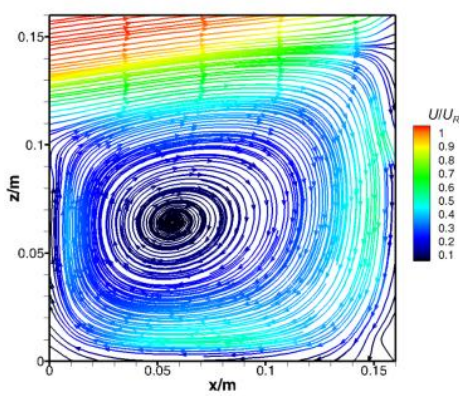

PIV measurement

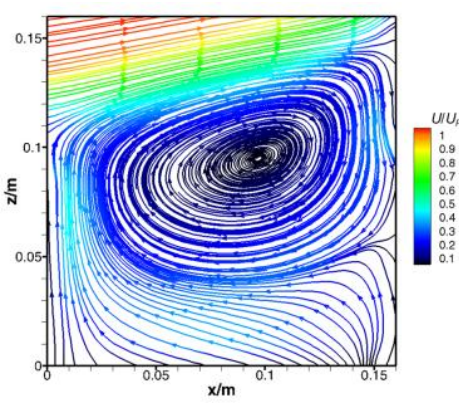

PIV measurement

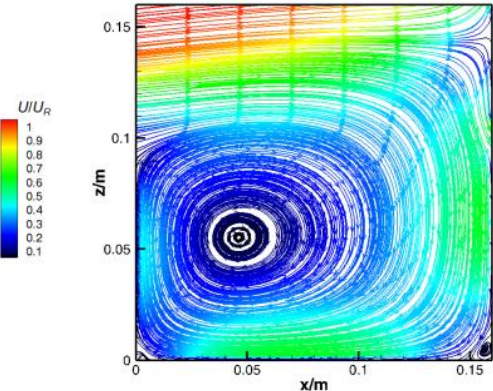

SKE-SWFs

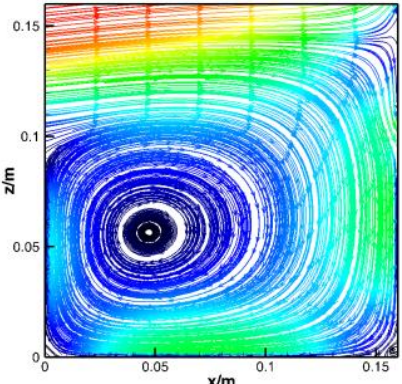

SKE-NEWFs

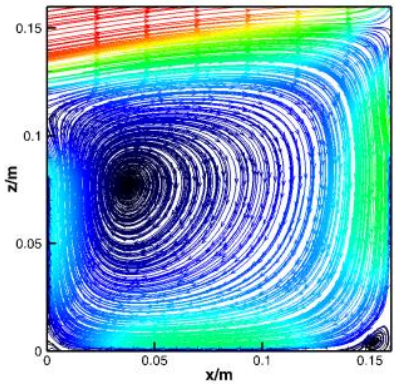

RLKE-SWFs

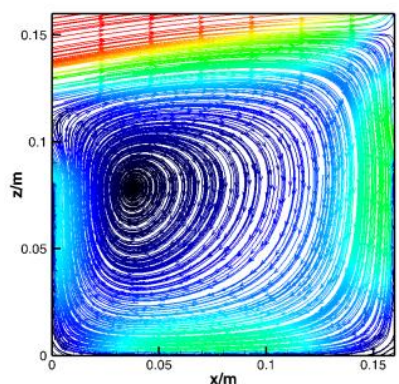

RLKE-NEWFs Case 1: $R i=0, R e=6.49 \mathrm{E}+04, G r=0$

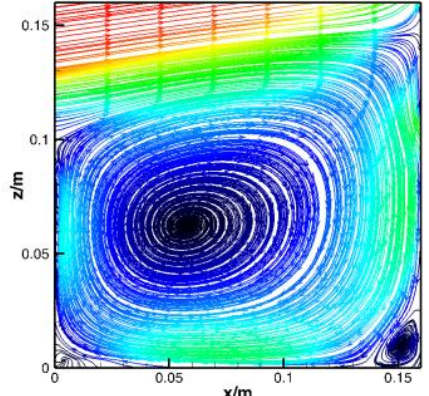

SKE-SWFs

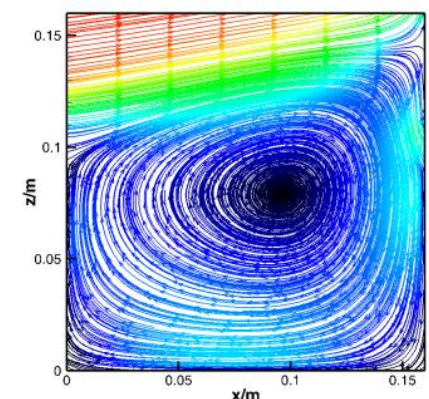

SKE-NEWFs

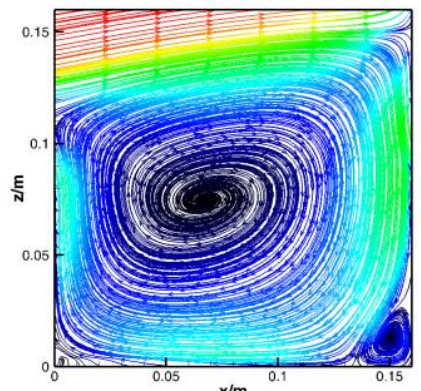

RLKE-SWFs

Case 4: $R i=0.85, R e=7.57 \mathrm{E}+03, G r=4.89 \mathrm{E}+07$

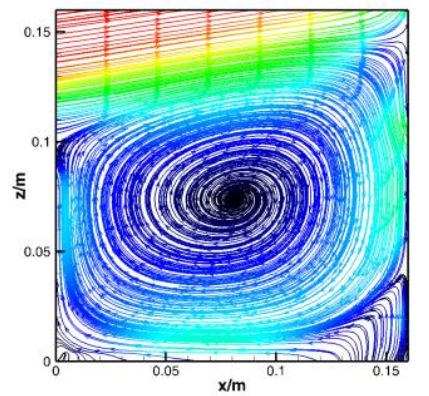

SKE-SWFs

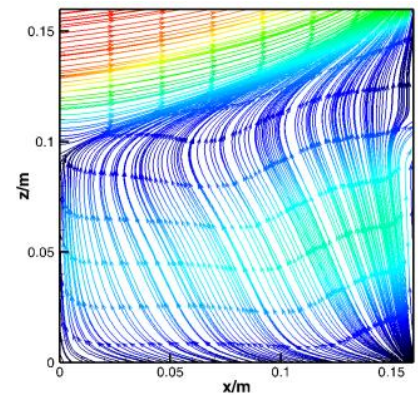

SKE-NEWFs

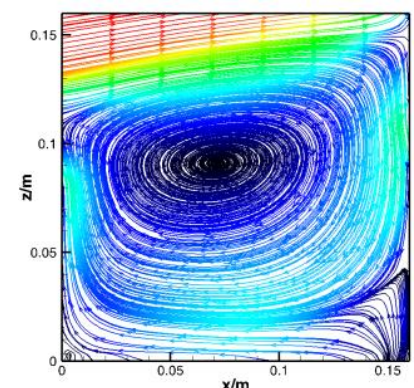

RLKE-SWFs

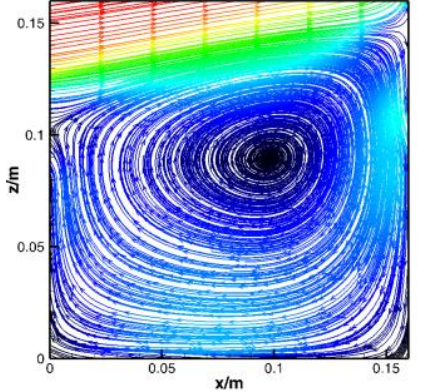

RLKE-NEWFs Case 5: $R i=1.22, R e=7.57 \mathrm{E}+03, G r=6.97 \mathrm{E}+07$

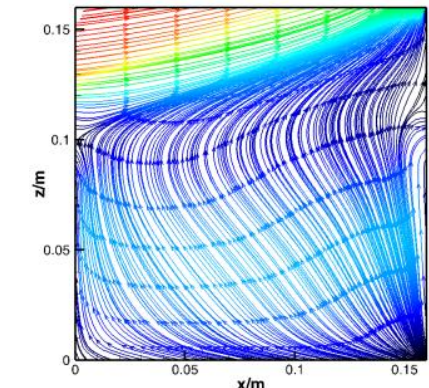

RLKE-NEWFs 


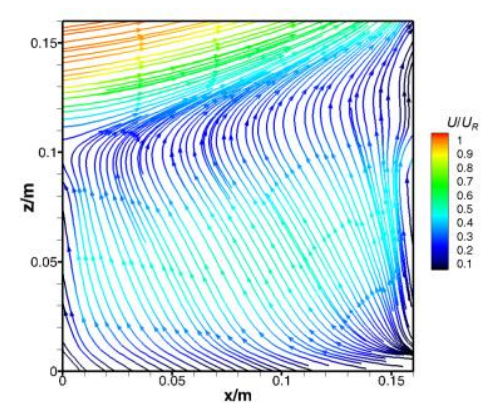

PIV measurement

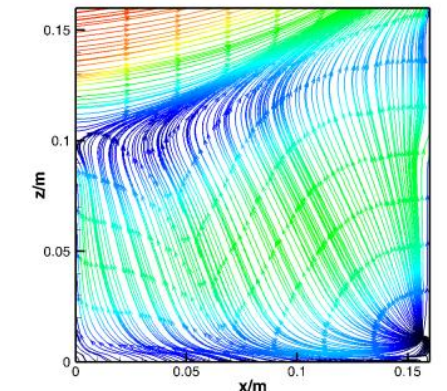

SKE-SWFs

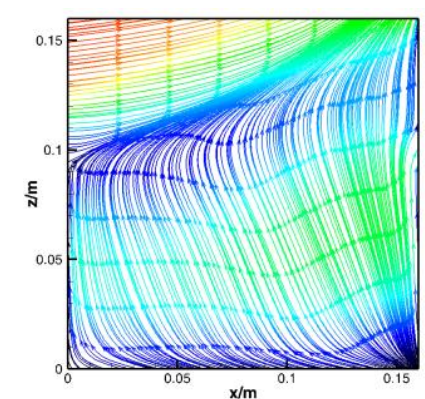

SKE-NEWFs

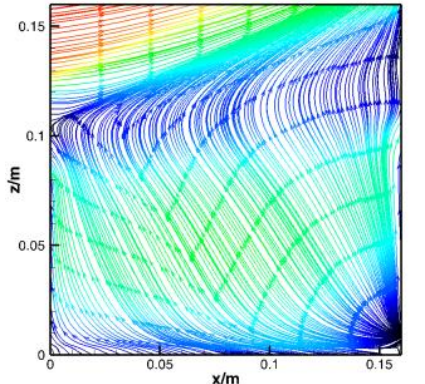

RLKE-SWFs

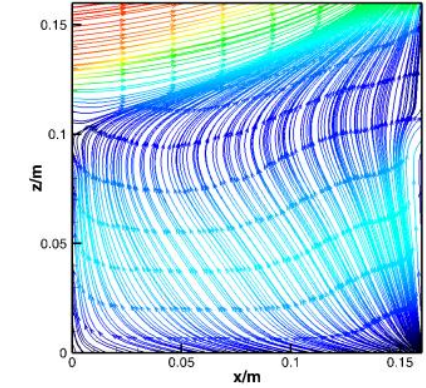

RLKE-NEWFs

Case 7: $R i=1.56, R e=7.57 \mathrm{E}+03, G r=8.93 \mathrm{E}+07$

Fig. 9. Normalized streamlines in the vertical central plane of street canyon for different cases. Wind tunnel measurements and CFD simulations by using four turbulence models (SKE-SWFs, SKE-NEWFs, RLKE-SWFs and RLKE-NEWFs). 


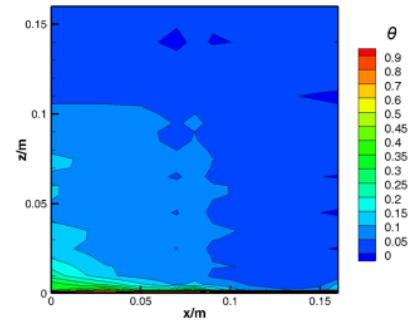

PIV measurement

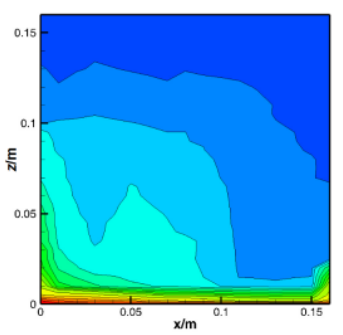

PIV measurement

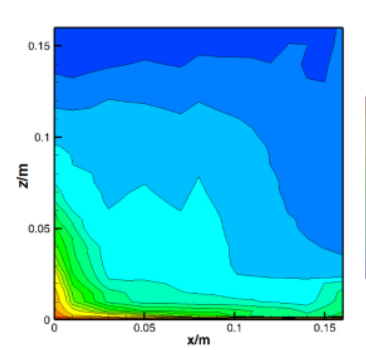

PIV measurement

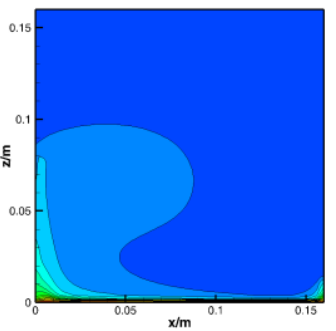

SKE-SWFs

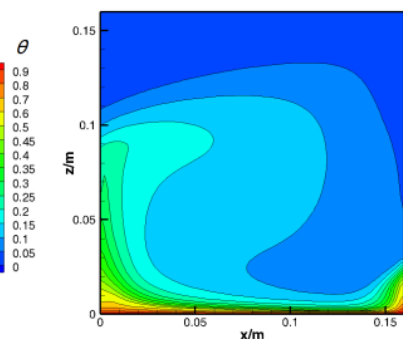

SKE-SWFs

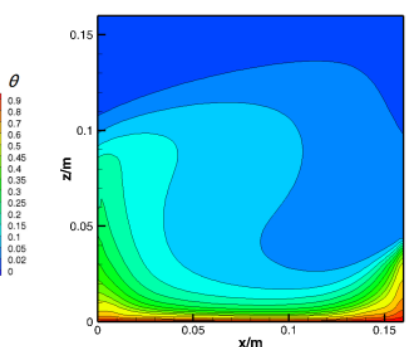

SKE-SWFs

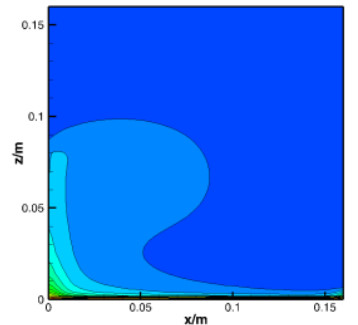

SKE-NEWFs

Case 2: $R i=0.01, R e=3.03 \mathrm{E}+04, G r=8.10 \mathrm{E}+06$

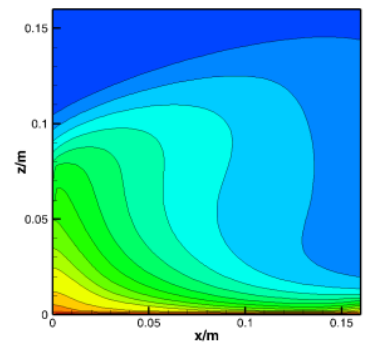

SKE-NEWFs$$
\text { RLKE-SWFS }
$$

Case 4: $R i=0.85, R e=7.57 \mathrm{E}+03, G r=4.89 \mathrm{E}+07$

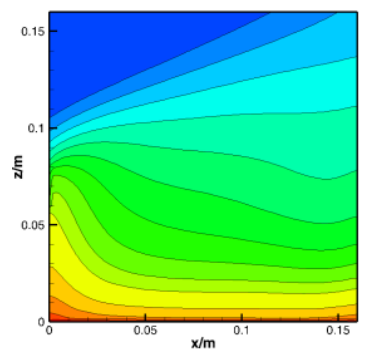

SKE-NEWFs

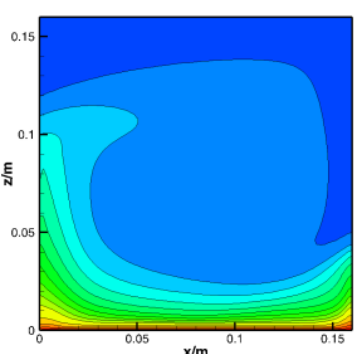

RLKE-SWFs
Case 5: $R i=1.22, R e=7.57 \mathrm{E}+03, G r=6.97 \mathrm{E}+07$

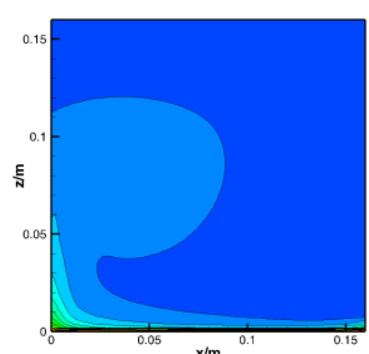

RLKE-NEWFs

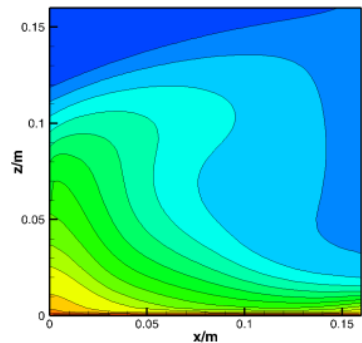

RLKE-NEWFs

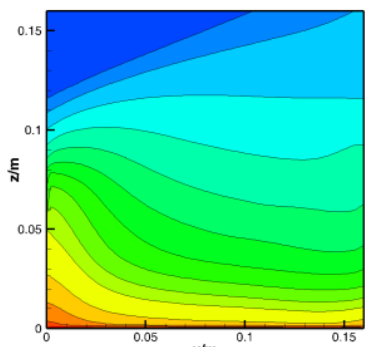

RLKE-NEWFs

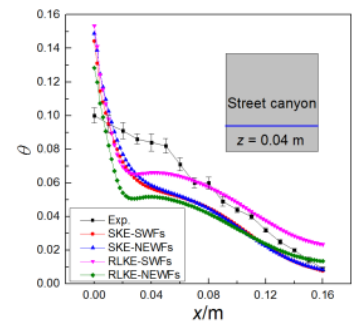

$\Theta$ along line $z=0.04 \mathrm{~m}$

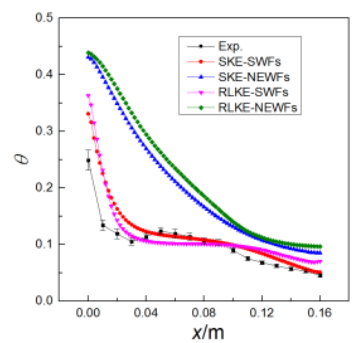

$\Theta$ along line $z=0.04 \mathrm{~m}$

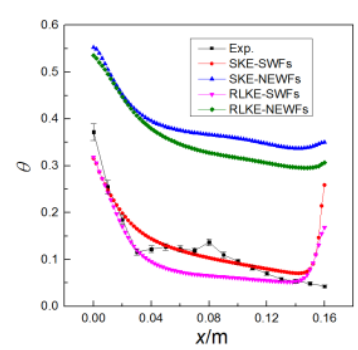

$\Theta$ along line $z=0.04 \mathrm{~m}$ 


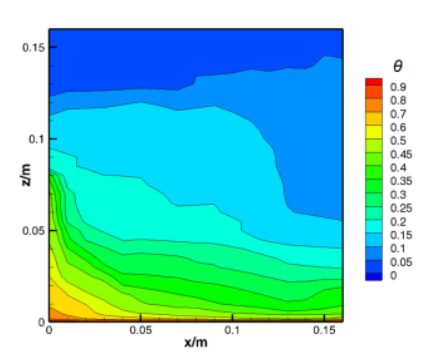

PIV measurement

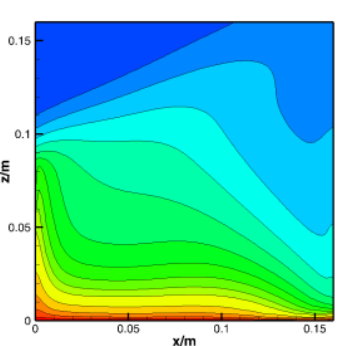

SKE-SWFs

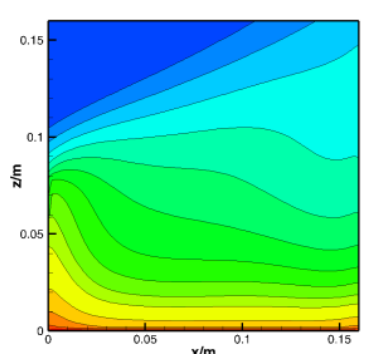

SKE-NEWFs

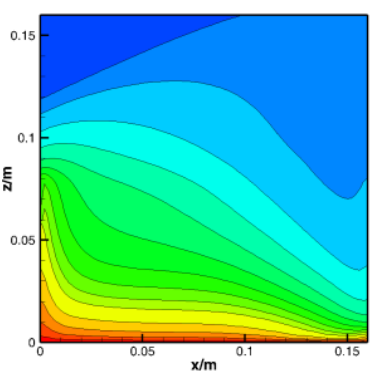

RLKE-SWFs

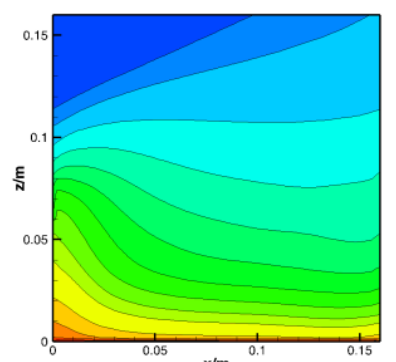

RLKE-NEWFs

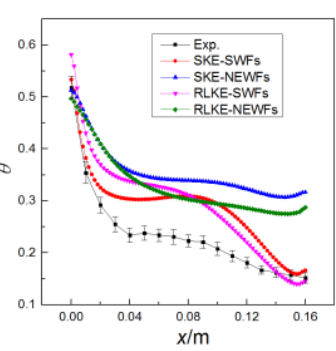

$\Theta$ along line $z=0.04 \mathrm{~m}$

Case 7: $R i=1.56, R e=7.57 \mathrm{E}+03, G r=8.93 \mathrm{E}+07$

Fig. 10. Distributions of dimensionless temperature $(\theta)$ in the vertical central plane of street canyon for different cases. Wind tunnel measurements and CFD simulations by using four turbulence models (SKE-SWFs, SKE-NEWFs, RLKE-SWFs and RLKE-NEWFs). 


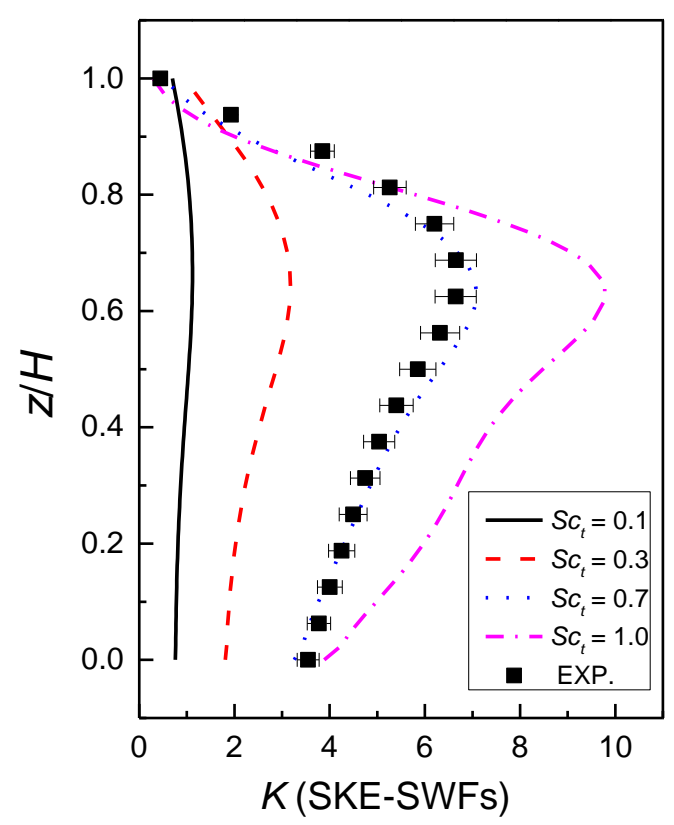

(a) Case 1: SKE-SWFs vs. EXP.

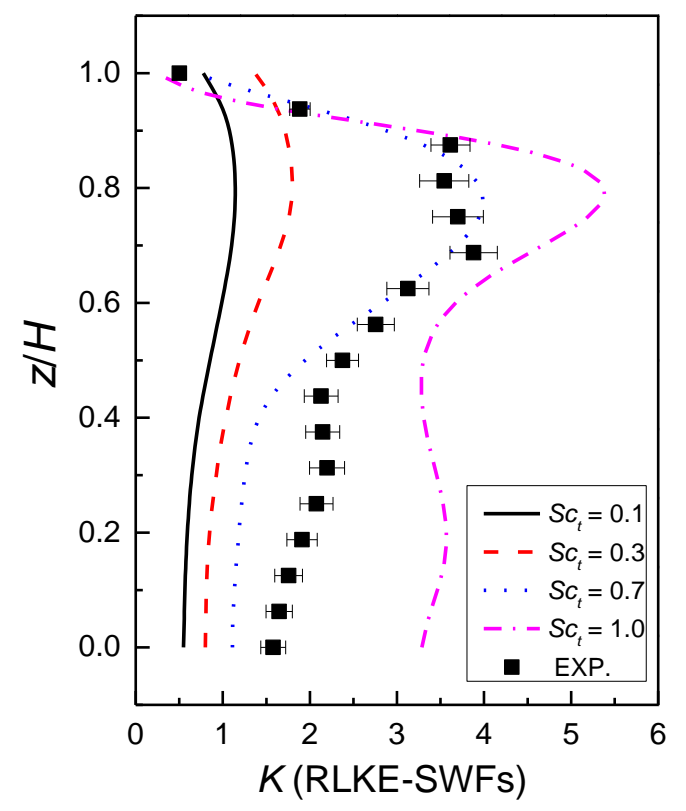

(b) Case 5: RLKE-SWFs vs. EXP.

Fig. 11. Normalized concentration distributions along the vertical centerline of the street canyon. Wind tunnel measurements and CFD simulations by using two turbulence models (SKE-SWFs and RLKE-SWFs) with different $S c_{t}$ numbers. 


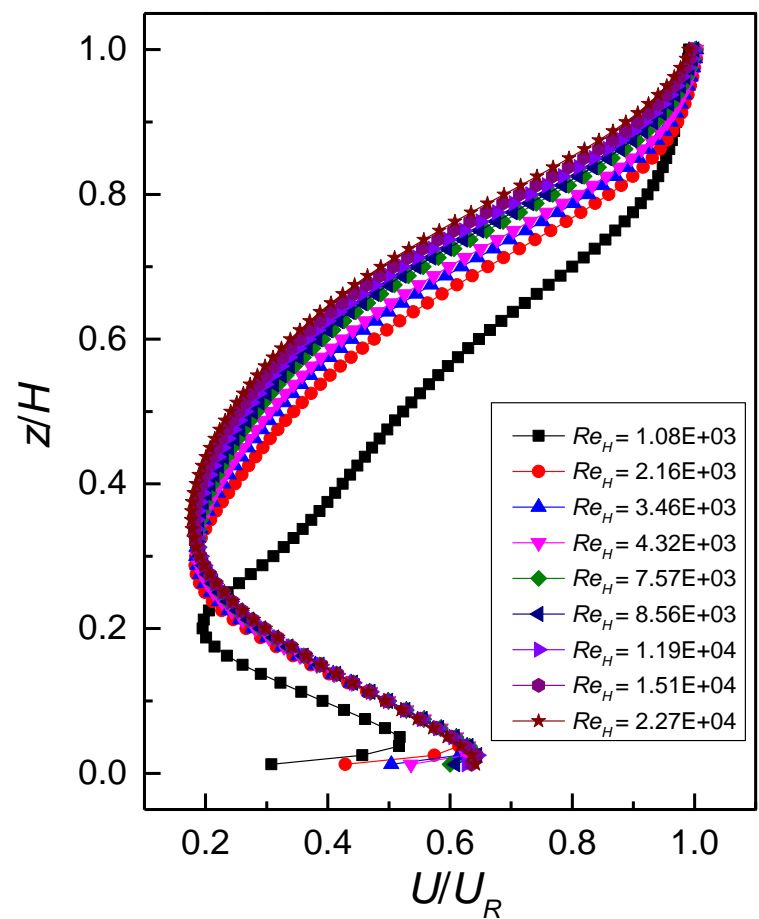

(a) Dimensionless velocity distributions along the vertical centerline of the street canyon with different street $R e_{H}$. 

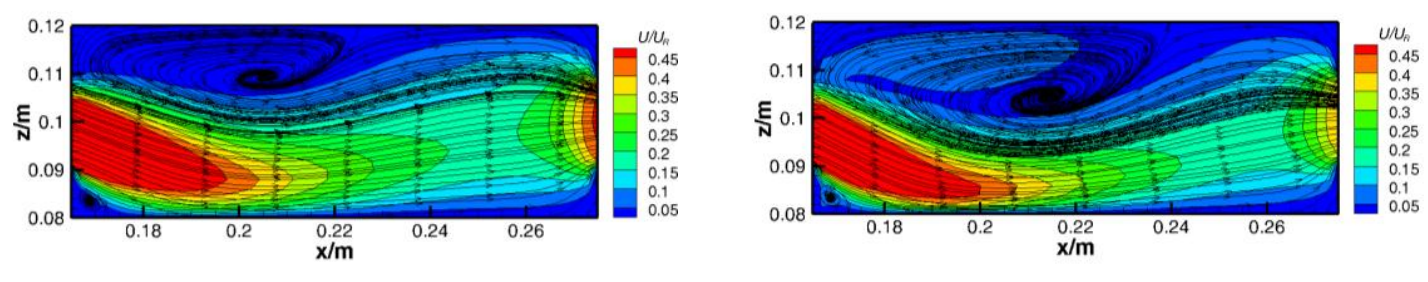

$R e_{H}=1.08 \mathrm{E}+03$

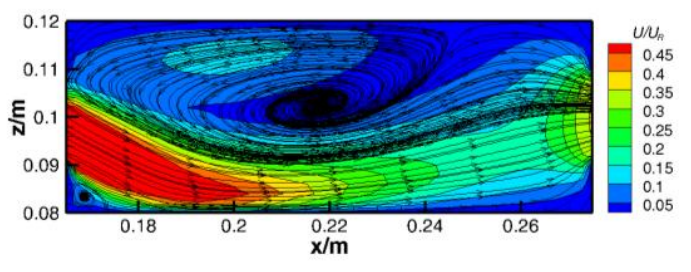

$R e_{H}=3.46 \mathrm{E}+03$

$R e_{H}=2.16 \mathrm{E}+03$

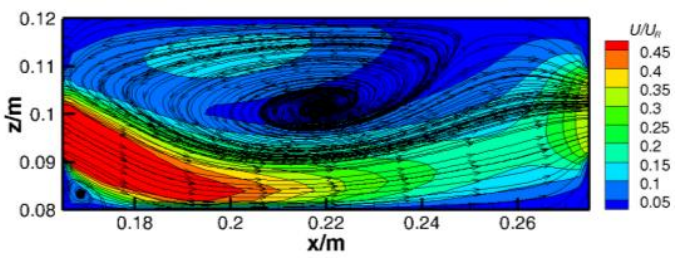

$R e_{H}=4.32 \mathrm{E}+03$
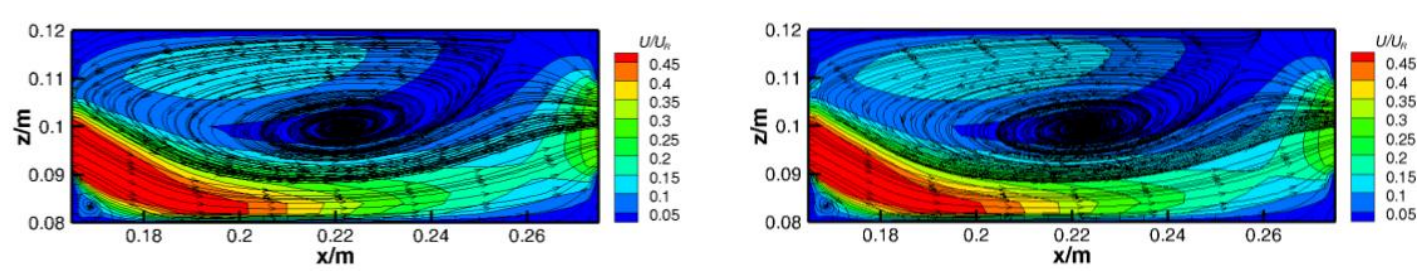

$R e_{H}=7.57 \mathrm{E}+03$

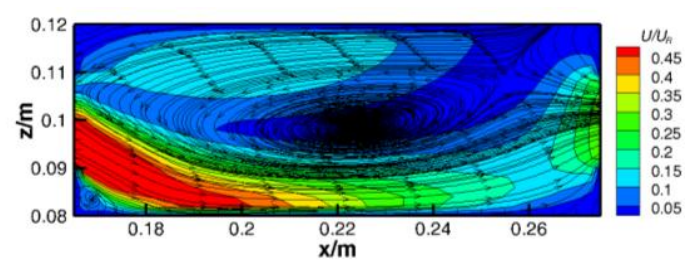

$R e_{H}=1.19 \mathrm{E}+04$

$R e_{H}=8.56 \mathrm{E}+03$

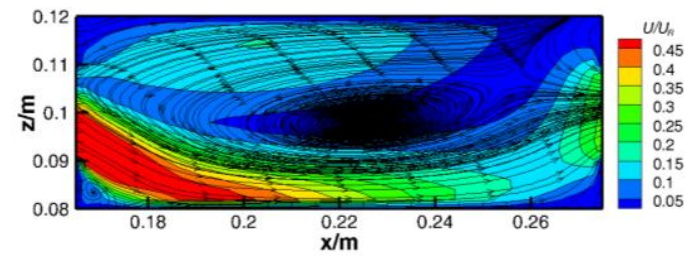

$R e_{H}=1.51 \mathrm{E}+04$

(b) Dimensionless velocity contours and streamlines in the room vertical center plane with different street $R e_{H}$.

Fig. 12. Flow structure independence analysis for the street scale and indoor scale models. 


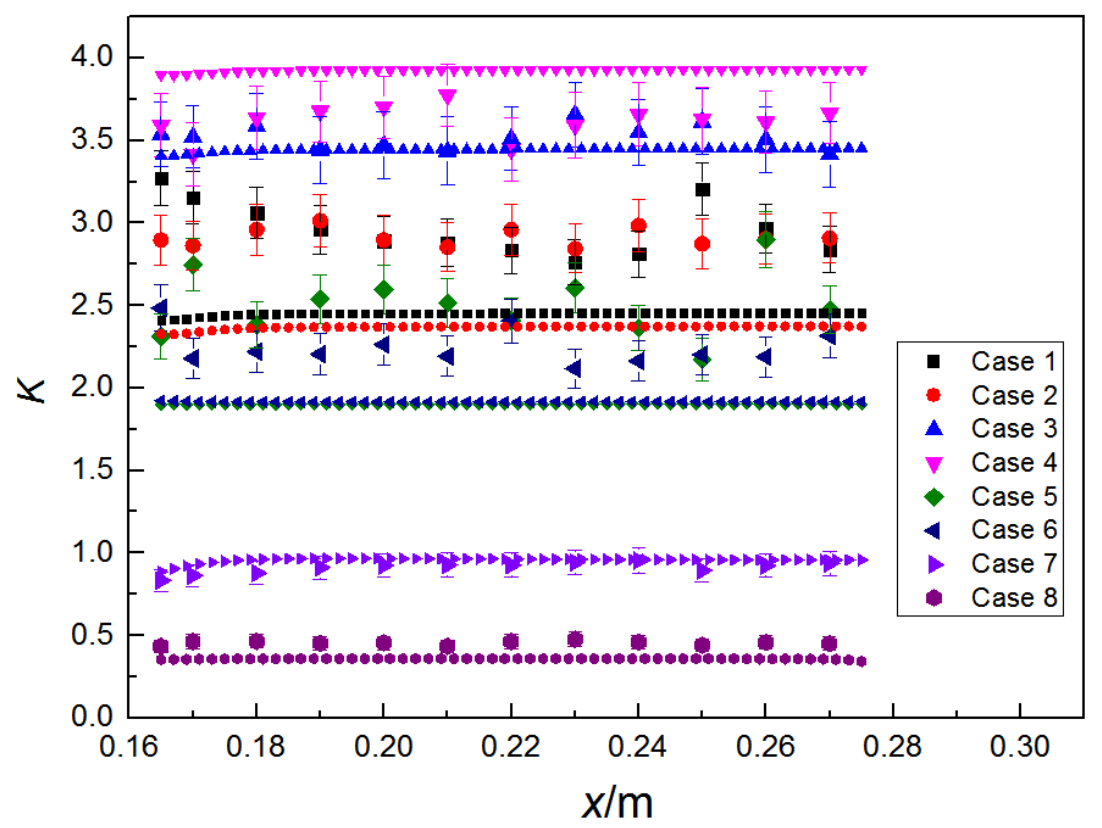

Fig. 13. Normalized pollutant distributions along the room horizontal center lines for different cases. Wind tunnel measurements (large symbols) and CFD simulations (small symbols). 